\title{
4-colouring of generalized signed planar graphs
}

\author{
Yiting Jiang Xuding Zhu* \\ Department of Mathematics \\ Zhejiang Normal University \\ Jinhua, China \\ $\{y t j i a n g, x d z h u\} @ z j n u . e d u . c n$
}

Submitted: Feb 2, 2020; Accepted: Jul 23, 2020; Published: Aug 21, 2020

(C) The authors. Released under the CC BY-ND license (International 4.0).

\begin{abstract}
Assume $G$ is a graph and $S$ is a set of permutations of positive integers. An $S$-signature of $G$ is a pair $(D, \sigma)$, where $D$ is an orientation of $G$ and $\sigma: E(D) \rightarrow S$ is a mapping which assigns to each $\operatorname{arc} e=(u, v)$ a permutation $\sigma(e)$ in $S$. We say $G$ is $S$ - $k$-colourable if for any $S$-signature $(D, \sigma)$ of $G$, there is a mapping $f: V(G) \rightarrow[k]$ such that for each $\operatorname{arc} e=(u, v)$ of $G, \sigma(e)(f(u)) \neq f(v)$. The concept of $S$ - $k$-colourable is a common generalization of many colouring concepts. This paper studies the problem as to which subsets $S$ of $S_{4}$, every planar graph is $S$-4-colourable. We call such a subset $S$ of $S_{4}$ a good subset. The Four Colour Theorem is equivalent to saying that $S=\{i d\}$ is good. It was proved by Jin, Wong and Zhu (arXiv:1811.08584) that a subset $S$ containing $i d$ is good if and only if $S=\{i d\}$. In this paper, we prove that, up to conjugation, every good subset of $S_{4}$ not containing $i d$ is a subset of $\{(12),(34),(12)(34)\}$.
\end{abstract}

Mathematics Subject Classifications: 05C10, 05C15

\section{Introduction}

A signed graph is a pair $(G, \sigma)$, where $G$ is a graph and $\sigma: E(G) \rightarrow\{1,-1\}$ is a mapping which assigns to each edge $e$ of $G$ a sign $\sigma(e) \in\{1,-1\}$. The mapping $\sigma$ is called a signature of $G$ and an edge $e$ is called positive or negative if $\sigma(e)=1$ or $\sigma(e)=-1$, respectively. Colouring of signed graphs was first studied by Zaslavsky [12] in 1980's, and has attracted a lot of recent attention. For a positive integer $k$, let $[k]=\{1,2, \ldots, k\}$ and let

$$
N_{k}= \begin{cases}\{ \pm 1, \pm 2, \ldots, \pm q\}, & \text { if } k=2 q \text { is even, } \\ \{0, \pm 1, \pm 2, \ldots, \pm q\}, & \text { if } k=2 q+1 \text { is odd }\end{cases}
$$

Let $Z_{k}=\{0,1, \ldots, k-1\}$ denote the cyclic group of order $k$.

\footnotetext{
${ }^{*}$ Supported by NSFC 11971438 and 111 project of Ministry of Education of China.
} 
Definition 1. Assume $(G, \sigma)$ is a signed graph and $k$ is a positive integer. A $k$-colouring of $(G, \sigma)$ is a mapping $f: V(G) \rightarrow N_{k}$ such that for any edge $e=x y$ of $G, f(x) \neq \sigma(e) f(y)$. A $Z_{k}$-colouring of $(G, \sigma)$ is a mapping $f: V(G) \rightarrow Z_{k}$ such that for any edge $e=x y$ of $G$, $f(x) \neq \sigma(e) f(y)$.

Note that for $i \in Z_{k},-i=k-i$ is the inverse of $i$ in group $Z_{k}$.

It is easy to see that if $k$ is odd, then a signed graph $(G, \sigma)$ is $k$-colourable if and only if it is $Z_{k}$-colourable, however, if $k$ is even, then $k$-colouring and $Z_{k}$-colouring are different. For example, if $G$ is an even cycle, then $(G, \sigma)$ is $Z_{2}$-colourable for any signature $\sigma$, however, if $G$ has an odd number of negative edges, then $(G, \sigma)$ is not 2-colourable.

Both $k$-colouring and $Z_{k}$-colouring of signed graphs are natural extensions of colouring of graphs and lead to interesting open problems. It was conjectured by Máčajová, Raspaud, Škoviera [8] that every signed planar graph is 4-colourable; and conjectured by Kang and Steffen [3] that every signed planar graph is $Z_{4}$-colourable. Both conjectures are stronger than the Four Colour Theorem. Recently, both conjectures are disproved. It was shown by Kardoš and Narboni [11] that there are signed planar graphs that are not 4-colourable, and shown by Zhu [14] that there are signed planar graphs that are not $Z_{4}$-colourable.

We are interested in signed planar graphs and hence we concentrate on colourings using 4 colours. There is another 4-colouring of signed planar graphs that is natural and interesting. We choose the colour set to be $\{1,-1, i,-i\}$, and define the Complex 4-colouring of a signed graph $(G, \sigma)$ as follows.

Definition 2 (Complex 4-colouring of signed graphs). A Complex 4-colouring of a signed graph $(G, \sigma)$ is a mapping $f: V(G) \rightarrow\{1,-1, i,-i\}$ such that for each edge $e=x y$, $f(x) f(y) \neq \sigma(e)$.

A natural question is whether every signed planar graph is Complex-4-colourable. We propose the following conjecture:

Conjecture 3. Every signed planar graph is Complex 4-colourable.

Unlike the conjecture of Máčajová, Raspaud, Škoviera and the conjecture of Kang and Steffen, Conjecture 3 is not stronger than the Four Colour Theorem. This feature makes this conjecture more appealing.

We shall explain in the next section, in some sense, Conjecture 3 is the only interesting Four Colouring Conjecture of signed planar graphs.

\section{Generalized signed graph colouring}

In colourings of a signed graph, the sign of an edge specify which colour pairs are forbidden to be assigned to its end vertices. It is natural to consider graphs with more variety of edges and hence with more types of restrictions on colour pairs that can be assigned to the end vertices of edges. Indeed, various colourings of graphs corresponding to different 
restrictions have been studied in the literature. Below are three types of graph colourings that are examples of such constraints.

In 1992, Jaeger, Linial, Payan and Tarsi [2] introduced the concept of group colouring. Assume $\Gamma$ is an Abelian group, $D$ is an orientation of $G$ and $\sigma: E(D) \rightarrow \Gamma$ assigns to each arc $e=(u, v)$ an element $\sigma(e) \in \Gamma$. A $\Gamma$-colouring of $(D, \sigma)$ is a mapping $f: V(G) \rightarrow \Gamma$ such that for any arc $e=(u, v)$ of $D, f(v)-f(u) \neq \sigma(e)$. We say $G$ is $\Gamma$-colourable if for any orientation $D$ of $G$ and for any $\sigma: E(D) \rightarrow \Gamma$, there exists a $\Gamma$-colouring of $(D, \sigma)$. The group chromatic number of $G$ is the minimum integer $k$ such that for any Abelian group $\Gamma$ of order $k, G$ is $\Gamma$-colourable ( see $[6,7,10]$ for some study on this subject).

In 1995, as a generalization of colouring of signed graphs, Zaslavsky [13] defined the colouring of gain graphs as follows: A gain graph consists of a graph $G$, a gain group $\Gamma$, and a gain function $\phi$, which assigns each orientation $e=(u, v)$ of an edge $u v$ a group element $\phi(e)$ so that $\phi\left(e^{-1}\right)=(\phi(e))^{-1}$, where $e^{-1}=(v, u)$ is the inverse of $e$. We denote a gain graph as $(G, \phi)$. A proper $k$-colouring of $(G, \phi)$ assigns to each vertex $v$ of $G$ a colour $f(v)$ from the colour set $\{0\} \cup\{(i, \pi): i \in\{1,2, \ldots, k\}, \pi \in \Gamma\}$, so that for each orientation $e=(u, v)$ of an edge $u v$ of $G$, if $f(u)=0$ then $f(v) \neq 0$, and if $f(u)=(i, \pi)$, then $f(v) \neq(i, \pi \circ \phi(e))$ (here $\circ$ is the product in $\Gamma)$. Note that if $\Gamma$ is an Abelian group, then a proper 1-colouring of $(G, \phi)$ without using colour 0 is the same as the $\Gamma$-colouring of $(G, \phi)$ defined as above by Jaeger, Linial, Payan and Tarsi [2]. If $\Gamma=Z_{2}$, then a $k$ colouirng of $(G, \phi)$ is a $(2 k+1)$-colouring of the signed graph $(G, \sigma)$, where $\sigma(e)=1$ if $\phi(e)=0$ and $\sigma(e)=-1$ if $\phi(e)=1$.

In 2018, Dvořák and Postle [1] defined DP-colouring of a graph as follows: Assume $G$ is a graph. A $k$-cover of $G$ is a graph $H$ with vertex set $V(G) \times[k]$ such that for each vertex $v,\{v\} \times[k]$ induces a clique, and for each edge $u v$ of $G$, edges between $\{v\} \times[k]$ and $\{u\} \times[k]$ is a matching, and there is no other edge. An independent set $I$ of $H$ with $|I|=|V(G)|$ is a DP-colouring of $G$ with respect to $H$. We say $G$ is DP-k-colourable if for any $k$-cover $H$ of $G$, there exists a DP-colouring of $G$ with respect to $H$. It was shown in [1] that every DP- $k$-colourable graph is $k$-choosable, and the concept of DP-colouring is used to show that every planar graph without cycles of lengths $4,5,6,7,8$ is 3-choosable, which is a problem that had remained open for 15 years.

In each of the colourings defined above, edges of $G$ are labeled, and certain pairs of colours are forbidden to be assigned to the end vertices of edges with given label. In this sense, the labels play the same role as the sign of the edges in a signed graph. However, instead of + and - signs, we may have many different signs. Adopting this point of view, we introduce the concept of colouring of $S$-signed graphs as follows.

A set $S$ of permutations of integers is called inverse closed if for each $\pi \in S, \pi^{-1} \in S$.

Definition 4. Assume $G$ is a graph and $S$ is an inverse closed set of permutations of positive integers. An $S$-signature of $G$ is a pair $(D, \sigma)$, where $D$ is an orientation of $G$ and $\sigma: E(D) \rightarrow S$ is a mapping which assigns to each $\operatorname{arc} e=(u, v)$ a permutation $\sigma(e) \in S$. A proper $k$-colouring of $(D, \sigma)$ is a mapping $f: V(D) \rightarrow[k]$ such that for each arc $e=(u, v)$ of $D, \sigma(e)(f(u)) \neq f(v)$. We say $G$ is $S$-k-colourable if every $S$-signature $(D, \sigma)$ of $G$ has a proper $k$-colouring. 
To define an $S$-signature of $G$, we need an orientation $D$ of $G$ and define a mapping $\sigma: E(D) \rightarrow S$. The orientation $D$ is just for reference. Indeed, if $E^{\prime}$ is a subset of $E(G)$ and $D^{\prime}$ is obtained from $D$ by reversing the orientations of edges in $E^{\prime}$, and

$$
\sigma^{\prime}(e)= \begin{cases}\sigma(e), & \text { if } e \notin E^{\prime}, \\ (\sigma(e))^{-1}, & \text { if } e \in E^{\prime},\end{cases}
$$

then a proper $k$-colouring of $(D, \sigma)$ is the same as a proper $k$-colouring of $\left(D^{\prime}, \sigma^{\prime}\right)$. In particular, if all the permutations $\pi \in S$ are involutions, i.e., $\pi^{-1}=\pi$, then the orientation of the edges are irrelevant, and we simply define a mapping $\sigma: E(G) \rightarrow S$.

The $k$-colouring and the $Z_{k}$-colouring of signed graphs are all colourings of $S$-signed graphs for some special sets $S$ of permutations. The sets $S$ consists of involutions only. Thus we do not need orientations of $G$.

Assume we are considering $S$ - $k$-colouring of a graph $G$. For $\pi \in S$, if $i \notin[k]$ or $\pi(i) \notin[k]$ and $e=(x, y)$ is an arc with $\sigma(e)=\pi$, then colouring $x$ with $i$ (or $\pi(i))$ puts no restriction on the colour of $y$. So for $i \notin[k]$ or $\pi(i) \notin[k]$, the image $\pi(i)$ is irrelevant. Sometimes it is convenient to allow permutations $\pi \in S$ to have $i \in[k]$ and $\pi(i) \notin[k]$ or $i \notin[k]$ and $\pi(i) \in[k]$. However, in this paper, when we consider $S$ - $k$-colourings of graphs, we are interested in those permutations $\pi$ with $\pi(i) \in[k]$ for every $i \in[k]$. In other words, the restriction of $\pi$ to $[k]$ is a permutation of $[k]$. As the images $\pi(i)$ of $i \notin[k]$ are irrelevant, we simply consider $\pi$ as a permutation of $[k]$, i.e., $S$ is an inverse closed subset of the symmetric group $S_{k}$.

We use $i d$ to denote the identity permutation. The other permutations of integers are written as product of cycles. For example, (12) is the permutation $\pi$ with $\pi(1)=2$, $\pi(2)=1$ and $\pi(i)=i$ for $i \neq 1,2 ;(12)(34)$ is the permutation $\pi$ with $\pi(1)=2, \pi(2)=1$, $\pi(3)=4, \pi(4)=3$ and $\pi(i)=i$ for $i \neq 1,2,3,4$.

The concept of colouring of generalized signed graphs is a common generalization of many colouring concepts:

Observation 5. Assume $S$ is a set of permutations of positive integers. Then the following hold:

- If $S=\{i d\}$, then $S$ - $k$-colourable is equivalent to $k$-colourable.

- If $S=\{i d,(12)(34) \ldots((2 q-1)(2 q))\}$ and $q=\lfloor k / 2\rfloor$, then $S$ - $k$-colourable is equivalent to signed $k$-colourable.

- If $S=\{i d,(12)(34) \ldots((2 q-1)(2 q))\}$ and $q=\lceil k / 2\rceil-1$, then $S$ - $k$-colourable is equivalent to signed $Z_{k}$-colourable.

- If $S=\{(12),(34)\}$, then $S$-4-colourable is equivalent to Complex 4-colourable.

- If $S=S_{k}$, then $S$ - $k$-colourable is equivalent to DP-k-colourable (also known as correspondence $k$-colourable) [1]. 
- If $\Gamma$ is an Abelian subgroup of $S_{k}$, then there is a subset $S$ of $S_{k}$ which is isomorphic to $\Gamma$ and $S$-colourable is equivalent to $\Gamma$-colourable in the sense defined by Jaeger et al [2]. For example, If $S$ is the subgroup of $S_{k}$ generated by $(12 \ldots k)$, then $S$ colourable is equivalent to $Z_{k}$-colourable, i.e., for any mapping $\phi: E(G) \rightarrow Z_{k}$, there is a mapping $f: V(G) \rightarrow Z_{k}$ such that for each arc $e=(x, y), f(y)-f(x) \neq \phi(e)$. We observe that $S$-k-colourable not only depends on the group structure of $S$, but depends on the permutations in $S$. It may happen that $S$ is a subgroup of $S_{k}$ isomorphic to $\Gamma$, but $S$ - $k$-colourable is different from $\Gamma$-colourable.

- Assume $\Gamma$ is a group with $|\Gamma|=n$ and $k$ is a positive integer. Let $\tau: \Gamma \rightarrow[n]$ be a one-to-one correspondence from $\Gamma$ to $[n]$. Let $k^{\prime}=k n+1$ and for each $\pi \in \Gamma$, let $\pi^{\prime}$ be the permutation of $\left[k^{\prime}\right]$ defined as $\pi^{\prime}(n j+r)=n j+\tau\left(\tau^{-1}(r) \circ \pi\right)$ for $r \in[n]$ and $j \in\{0,1, \ldots, k-1\}$, and $\pi^{\prime}(k n+1)=k n+1$. Let $S=\left\{\pi^{\prime}: \pi \in \Gamma\right\}$. Then $a$ $k$-colouring of a gain graph $(G, \phi)$ with gain group $\Gamma$ is equivalent to a $k^{\prime}$-colouring of an $S$-signature $(D, \sigma)$ of $G$, where $\sigma$ is defined as $\sigma(e)=\pi^{\prime}$ if $\phi(e)=\pi$.

- A signature $\sigma$ of a graph $G$ is called $k$-consistent if for every directed cycle $C=$ $\left(e_{1}, e_{2}, \ldots, e_{k}\right)$ of $G$, the composition $\pi=\sigma\left(e_{1}\right) \sigma\left(e_{2}\right) \ldots \sigma\left(e_{k}\right)$ of permutations on the edges of the cycle has the following property: for $i \in[k]$, either $\pi(i)=i$ or for some $1 \leq j \leq k, \sigma\left(e_{1}\right) \sigma\left(e_{2}\right) \ldots \sigma\left(e_{j}\right)(i) \notin[k]$. A graph $G$ is $k$-choosable if and only if $(G, \sigma)$ is $k$-colourable for every $k$-consistent signature $\sigma$ of $G$. Here it is necessary to consider permutations $\pi$ whose restriction of $[k]$ is a partial permutation, i.e., it is allowed that $\pi(i) \notin[k]$ for $i \in[k]$.

Proof. All the statements above follow straightforward from the definition. We give a brief explanation of the last one.

Assume $(G, \sigma)$ is $k$-colourable for every $k$-consistent signature $\sigma$ of $G$, and $L$ is a $k$ list assignment of $G$. Without loss of generality, we may assume that colours in $L(v)$ for $v \in V(G)$ are positive integers. For each vertex $v$, let $\pi_{v}$ be any permutation of positive integers such that $\pi_{v}([k])=L(v)$. For each arc $e=(x, y)$ of $G$, let $\sigma(e)(i)=j$ if $\pi_{x}(i)=\pi_{y}(j)$ and $\sigma(e)(i) \notin[k]$ if $\pi_{x}(i) \notin L(y)$. Assume $i \in[k], C=\left(e_{1}, e_{2}, \ldots, e_{k}\right)$ is a cycle, and for $1 \leq j \leq k, \sigma\left(e_{1}\right) \sigma\left(e_{2}\right) \ldots \sigma\left(e_{j}\right)(i) \notin[k]$. Assume $e_{j}=x_{j} x_{j+1}$, where $x_{k+1}=x_{1}$. If $\sigma\left(e_{1}\right) \sigma\left(e_{2}\right) \ldots \sigma\left(e_{k}\right)(i)=i^{\prime}$, then it follows from the definition that $\pi_{x_{1}}(i)=\pi_{x_{1}}\left(i^{\prime}\right)$. Since $\pi_{x_{1}}$ is a permutation of integers, we conclude that $i^{\prime}=i$. So $\sigma$ is a $k$-consistent $S$-signature of $G$. Hence $(G, \sigma)$ has a $k$-colouring $\phi$. Let $\psi(v)=\pi_{v}(\phi(v))$. Then it is easy to verify that $\psi$ is an $L$-colouring of $G$.

Assume $G$ is $k$-choosable and $\sigma$ is $k$-consistent signature of $G$. We build a graph $H$ with vertex set $V(G) \times[k]$, in which $(x, i)(y, j)$ is an edge of $H$ if and only if $e=(x, y) \in E(G)$ and $\sigma(e)(i)=j$. Since $\sigma$ is $k$-consistent, for each vertex $x$ of $G$, each connected component $C$ of $H$ contains at most one vertex from $\{x\} \times[k]$. Let $C_{1}, C_{2}, \ldots, C_{p}$ be the connected components of $H$. For each vertex $x$ of $G$, let $L(x)=\left\{i: C_{i}\right.$ contains one vertex from $\{x\} \times[k]\}$. Then $L$ is a $k$-list assignment of $G$. By assumption, there is an $L$-colouring $\phi$ of $G$. For each vertex $x$ of $G$, let $\psi(x)=i$ if $\phi(x)=j$ and $C_{j}$ contains $(x, i)$. It is straightforward to verify that $\psi$ is a $k$-colouring of $(G, \sigma)$. 
In the following, we concentrate on $S$ that are subsets of $S_{k}$.

Observe that if $S$ is a subset of $S_{k}$ and there is an integer $a \in[k]$ such that for any $\pi \in S, \pi(a) \neq a$, then for any $S$-signed graph $(G, \sigma)$, the mapping $f(v)=a$ for all $v \in V(G)$ is a $k$-colouring of $(G, \sigma)$.

Definition 6. We call a subset $S$ of $S_{k}$ normal if for each $a \in[k]$, there is a permutation $\pi \in S$ such that $\pi(a)=a$.

By the observation above, we shall restrict to normal subsets of $S_{k}$. One particular type of normal subsets $S$ of $S_{k}$ consists of those subsets $S$ containing $i d$.

In this paper, we concentrate on 4-colouring of generalized signed planar graphs. We are interested in the following question.

Question 7. For which subsets $S$ of $S_{4}$, every planar graph is $S$-4-colourable?

We call a subset $S$ of $S_{4}$ good if every planar graph is $S$-colourable. The Four Colour Theorem says that $S=\{i d\}$ is good.

Definition 8. We say two subsets $S$ and $S^{\prime}$ of $S_{k}$ are conjugates, if there is a permutation $\pi \in S_{k}$ such that $S^{\prime}=\left\{\pi \sigma \pi^{-1}: \sigma \in S\right\}$.

It is obvious that if $S$ and $S^{\prime}$ are conjugates and $S$ is good, then $S^{\prime}$ is good. The following result was proved in [3].

Theorem 9. [3] If $S$ is a good subset of $S_{4}$ containing id, then $S=\{i d\}$.

In this paper, we prove the following result.

Theorem 10. Up to conjugation, every good subset of $S_{4}$ not containing id is a subset of $\{(12),(34),(12)(34)\}$.

As a consequence of Theorem 10 and Theorem 9, the question whether $S$ is a good subset of $S_{4}$ remains open only for $S=\{(12),(34),(12)(34)\}$ and $S=\{(12),(34)\}$. Moreover, $\{(12),(34)\}$ is good if and only if every signed planar graph is Complex 4-colourable.

\section{Proof of Theorem 10}

To prove Theorem 10, we shall show that if $S$ is a normal subset of $S_{4}$ not containing id and $S$ is not conjugate to a subset of $\{(12),(34),(12)(34)\}$, then there is a planar graph $G$ which is not $S$-4-colourable.

For convenience, the sets $S$ listed in this section need not be inverse closed. If $\pi \neq \pi^{-1}$ and $\pi \in S$, then $\pi^{-1}$ is implicitly assumed to be a member of $S$, but will not be listed explicitly as a member of $S$.

Definition 11. Assume $S$ is a normal subset of $S_{4}$. A subset $S^{\prime}$ of $S$ is called a core of $S$ if $S^{\prime}$ is a minimal normal subset of $S$. 
For example, the set $\{i d,(12),(34),(12)(34)\}$ has two cores: $\{i d\},\{(12),(34)\}$.

Lemma 12. Assume $S$ is a normal subset of $S_{4}$ and $i d \notin S$. Then up to conjugation, $S$ has a core that is one of the following sets: $\{(12),(34)\},\{(12),(13),(23)\},\{(12),(13),(234)\}$, $\{(12),(134),(234)\},\{(234),(134),(124),(123)\}$.

Proof. Assume $S$ is a minimal normal subset of $S_{4}$ not containing $i d$. Then each permutation in $S$ fixes one or two colours in [4]. So $2 \leq|S| \leq 4$.

If $|S|=2$, then each permutation in $S$ fixes exactly two colours in [4]. In this case, up to conjugation, $S=\{(12),(34)\}$.

If $|S|=3$, then one permutation in $S$ fixes two colours in [4]. Without loss of generality, we may assume that $(12) \in S$. Since $S$ is a minimal normal subset of $S_{4}$, no permutation in $S$ fixes both 1 and 2 . So one of the remaining permutation $\pi_{1} \in$ $S$ fixes 1, and the other permutation $\pi_{2} \in S$ fixes 2. Thus $\pi_{1} \in\{(23),(24),(234)\}$ and $\pi_{2} \in\{(13),(14),(134)\}$. Thus up to conjugation, $S$ is one of the following sets: $\{(12),(13),(23)\},\{(12),(13),(234)\},\{(12),(134),(234)\}$.

If $|S|=4$, then each permutation in $S$ fixes exactly one colour in [4]. So $S=$ $\{(234),(134),(124),(123)\}$.

Lemma 13. Let $\mathscr{S}=$

$\{\{(12),(34),(123)\},\{(12),(34),(1234)\},\{(12),(34),(2314)\},\{(12),(34),(23)\},\{(12)$, $(34),(23)(14)\},\{(12),(13),(23)\},\{(12),(13),(234)\},\{(12),(134),(234)\},\{(234),(134)$, $(124),(123)\}\}$. If $S$ is a normal subset of $S_{4}$ not containing id and $S$ is not conjugate to a subset of $\{(12),(34),(12)(34)\}$, then $S$ has a subset which is a conjugation of a member of $\mathscr{S}$.

Proof. If $\{(12),(34)\}$ is a core of $S$, then $S$ contains another permutation $\pi$ such that $\pi \neq$ $i d$ and $\pi \neq(12)(34)$. So up to conjugation, $S \in\{\{(12),(34),(123)\},\{(12),(34),(1234)\}$, $\{(12),(34),(2314)\},\{(12),(34),(23)\},\{(12),(34),(23)(14)\}\}$.

If $\{(12),(34)\}$ is not a core of $S$ (up to conjugation), then by Lemma 12 , the core of $S$ is already conjugate to a member of $\mathscr{S}$.

Thus to prove Theorem 10, it suffices to show that for each $S \in \mathscr{S}$, there is a planar graph $G$ and an $S$-signature $(D, \sigma)$ of $G$ such that $(D, \sigma)$ is not 4-colourable.

In the remainder of this paper, let $\pi^{*}=(12)$.

Lemma 14. For any $S \in \mathscr{S}$, for any $a, b \in[4]$ for which $\pi^{*}(a) \neq b$ (it is possible that $a=b)$, there is a planar graph $H$ with $u, u^{\prime}$ be two vertices on the boundary of $H$, such that there is an $S$-signature $(D, \sigma)$ of $H$ and there is no 4-colouring $\phi$ of $(D, \sigma)$ with $\phi(u)=a$ and $\phi\left(u^{\prime}\right)=b$.

We leave the proof of Lemma 14 to the next section. With this lemma, we can complete the proof of Theorem 10.

Assume $S \in \mathscr{S}$. For each pair $(a, b)$ of integers with $a, b \in[4]$ and $\pi^{*}(a) \neq b$, let $H_{a, b}$ be a planar graph with $u, u^{\prime}$ be two vertices on the boundary of $H_{a, b}$, such that there 
is an $S$-signature $\sigma$ of $H_{a, b}$ and there is no 4-colouring $\phi$ of $\left(H_{a, b}, \sigma\right)$ with $\phi(u)=a$ and $\phi\left(u^{\prime}\right)=b$.

Let $G$ be obtained from the disjoint union of $\left\{H_{a, b}: a, b \in[4], \pi^{*}(a) \neq b\right\}$ by identifying all the copies of $u$ into a single vertex $u^{*}$, all the copies of $u^{\prime}$ into a single vertex $v^{*}$ and adding an edge $u^{*} v^{*}$.

If $(12) \in S$, then let $(D, \sigma)$ be the $S$-signature of $G$ whose restriction to each $H_{a, b}$ is as defined above and $\sigma\left(u^{*} v^{*}\right)=\pi^{*}$ (note that as $\left(\pi^{*}\right)^{-1}=\pi^{*}$, the orientation of the edge $u^{*} v^{*}$ is irrelevant). It follows that there is no 4-colouring of $(D, \sigma)$. Indeed, if $\phi$ is a 4-colouring of $G$ with $\phi(u)=a$ and $\phi(v)=b$, then since $\sigma\left(u^{*} v^{*}\right)=\pi^{*}$, we have $\pi^{*}(a) \neq b$. Then by Lemma 14, the restriction of $\phi$ to $H_{a, b}$ is not a proper 4-colouring of $H_{a, b}$.

If (12) $\notin S$, i.e., $S=\{(234),(134),(124),(123)\}$, then let $G^{\prime}$ be obtained from the disjoint union of three copies of $G$ by identifying the three copies of $u^{*}$ into a single vertex, which is still named $u^{*}$. Let $(D, \sigma)$ be the $S$-signature of $G$ whose restriction to each $H_{a, b}$ is as defined above and in the first copy of $G$, the edge $u^{*} v^{*}$ is oriented as $\left(u^{*}, v^{*}\right)$ and $\sigma\left(u^{*}, v^{*}\right)=(123)$. In the second copy of $G^{*}$, the edge $u^{*} v^{*}$ is oriented as $\left(v^{*}, u^{*}\right)$ and $\sigma\left(v^{*}, u^{*}\right)=(123)$. In the third copy of $G^{*}$, the edge $u^{*} v^{*}$ is oriented as $\left(v^{*}, u^{*}\right)$ and $\sigma\left(v^{*}, u^{*}\right)=(124)$. Now we show that there is no 4-colouring of $(D, \sigma)$. Assume to the contrary that $\phi$ is a proper 4 -colouring of $(D, \sigma)$. If $\phi\left(u^{*}\right)=1$, then $\phi\left(v^{*}\right) \neq 2$ for $v^{*}$ in the first copy of $G$. The same argument as above leads to a contradiction. If $\phi\left(u^{*}\right)=2$, then $\phi\left(v^{*}\right) \neq 1$ in the second copy of $G$. Similarly, we arrive at a contradiction. If $\phi\left(u^{*}\right)=3$, then $\phi\left(v^{*}\right) \neq 3$ in the third copy of $G$. Again a contradiction. If $\phi\left(u^{*}\right)=4$, then $\phi\left(v^{*}\right) \neq 4$ in the first copy of $G$, which also leads to a contradiction. This completes the proof of Theorem 10.

\section{Proof of Lemma 14}

For different sets $S \in \mathscr{S}$, the constructions of $H$ and the $S$-signature $(D, \sigma)$ are different, but some of them are very similar to each other. By grouping similar constructions together, we divide the proof of Lemma 14 into four claims.

The construction will use five graphs $H_{1}, H_{2}, H_{3}, H_{4}, H_{5}$ as gadgets. These five graphs are depicted in Figures 1 and 2, where in Figure 2, each 3-face of $H_{5}$ containing a * contains a copy of $T$ whose boundary triangle is identified with the 3 -face.

Claim 15. If $S \in\{\{(12),(34),(1234)\},\{(12),(34),(2314)\},\{(12),(34),(23)(14)\},\{(12)$, $(34),(23)\},\{(12),(13),(234)\},\{(12),(134),(234)\}\}$, then for any $a, b \in[4]$ with $\pi^{*}(a) \neq b$, there is an $S$-signature $(D, \sigma)$ of $H_{1}$ such that there is no 4-colouring $\phi$ of $\left(H_{1}, \sigma\right)$ with $\phi(u)=a$ and $\phi\left(u^{\prime}\right)=b$.

Proof. We consider three cases.

Case 1. $S \in\{\{(12),(34),(1234)\},\{(12),(34),(2314)\},\{(12),(34),(23)(14)\}\}$.

By symmetry, it suffices to consider the cases that $a=b=1$ or $a=1, b=3$ or $a=1, b=4$ or $a=3, b=4$.

First we assume $S=\{(12),(34),(1234)\}$. 

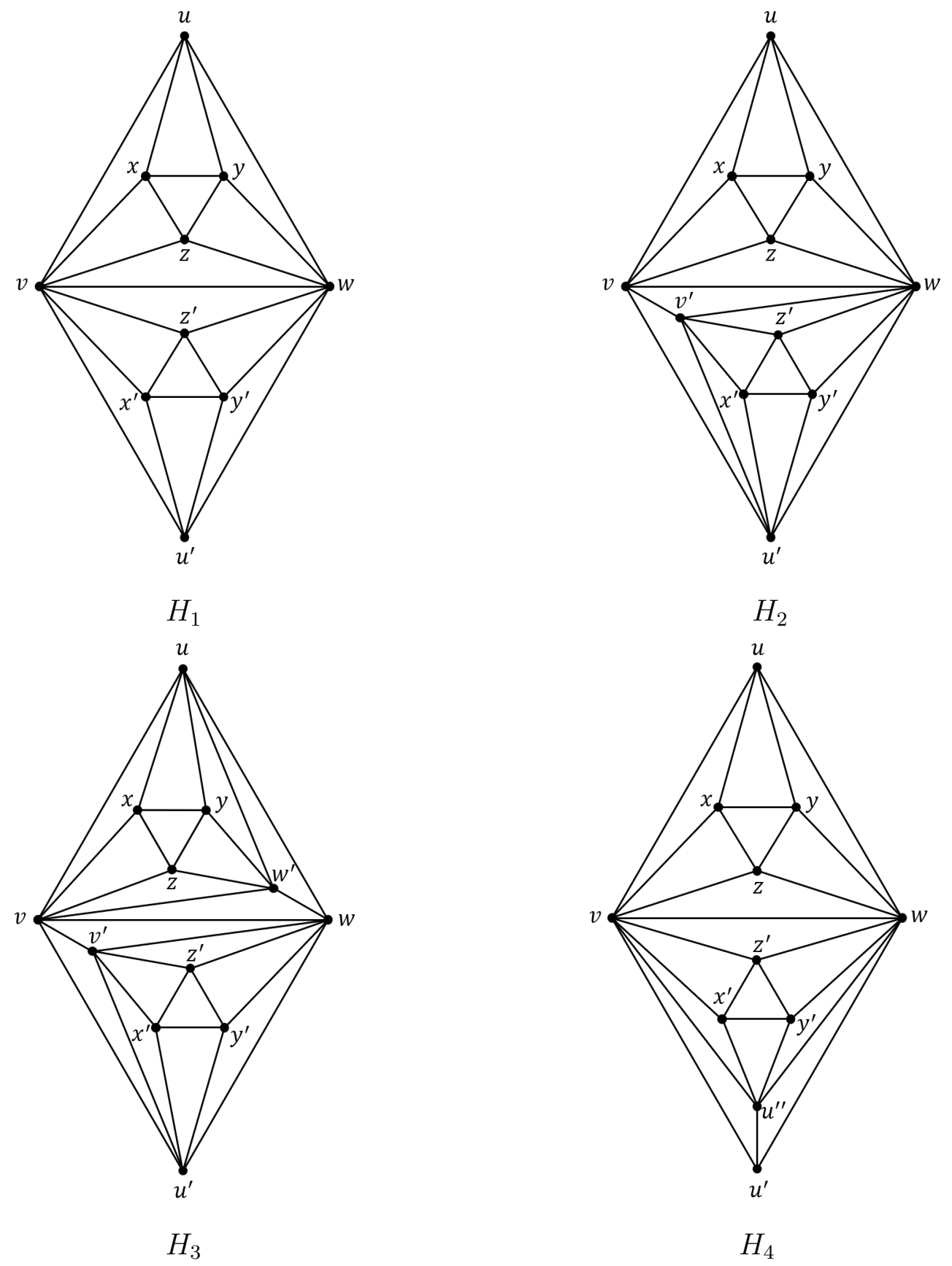

Figure 1: The graphs $H_{1}, H_{2}, H_{3}, H_{4}$ 


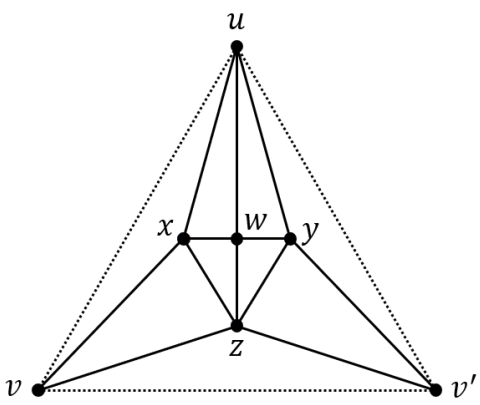

$T$

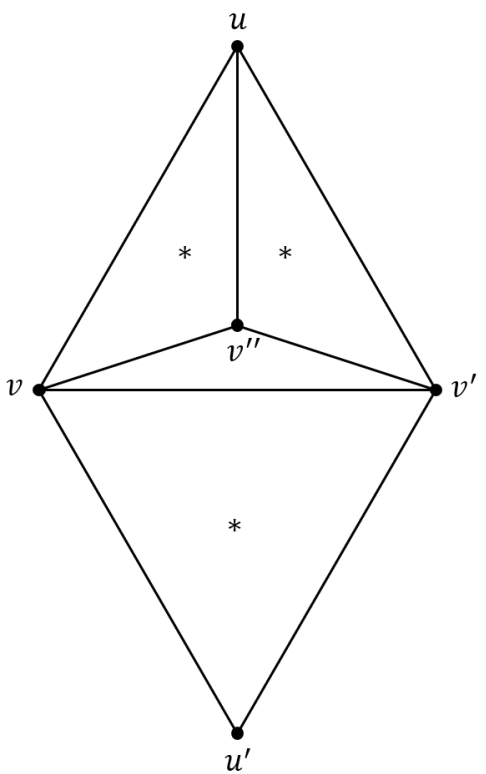

$H_{5}$

Figure 2: The graphs $T$ and $H_{5}$

- If $a=b=1$, then let $(D, \sigma)$ be the $S$-signature of $H_{1}$ defined as in Figure 3(a).

- If $a=1, b=3$, then let $(D, \sigma)$ be the $S$-signature of $H_{1}$ defined as in Figure 3(b).

- If $a=1, b=4$, then let $(D, \sigma)$ be the $S$-signature of $H_{1}$ defined as in Figure 3(c).

- If $a=3, b=4$, then let $(D, \sigma)$ be the $S$-signature of $H_{1}$ defined as in Figure 3(d).

Consider Figure 3(a). Assume to the contrary that $\phi$ is a 4-colouring of $\left(H_{1}, \sigma\right)$ with $\phi(u)=\phi\left(u^{\prime}\right)=1$. The edge $e=u v$ has $\sigma(e)=(12)$. This implies that $\phi(v) \neq 2$. The edge $e=u^{\prime} v$ has $\sigma(e)=(34)$. This implies that $\phi(v) \neq 1$. So $\phi(v) \in\{3,4\}$. For the same reason, $\phi(w) \in\{3,4\}$.

Since $e=v w$ has $\sigma(e)=(12)$, we conclude that $\phi(v) \neq \phi(w)$.

Assume first that $\phi(v)=3$ and $\phi(w)=4$.

Then none of $x, y, z$ can be coloured by 3 or 4 . So $x, y, z$ are all coloured by 1 and 2 . However, the three edges $e$ connecting $x, y, z$ have $\sigma(e)=(34)$. Hence no two vertices of $x, y, z$ can be coloured by the same colour, a contradiction.

If $\phi(v)=4$ and $\phi(w)=3$, then $x^{\prime}, y^{\prime}, z^{\prime}$ are all coloured by 1 and 2 , and no two vertices of $x^{\prime}, y^{\prime}, z^{\prime}$ can be coloured by the same colour, a contradiction.

Figure $3(\mathrm{~b})$ is checked similarly.

Assume $(D, \sigma)$ is defined by Figure $3(\mathrm{~b})$ and $\phi$ is a 4 -colouring of $\left(H_{1}, \sigma\right)$ with $\phi(u)=1$ and $\phi\left(u^{\prime}\right)=3$. Then by checking the permutations assigned to edges between $\left\{u, u^{\prime}\right\}$ and $\{v, w\}$, we conclude that $(\phi(v), \phi(w))=(3,4)$ or $(4,3)$. Depending on whether $\phi(v)=3$ 


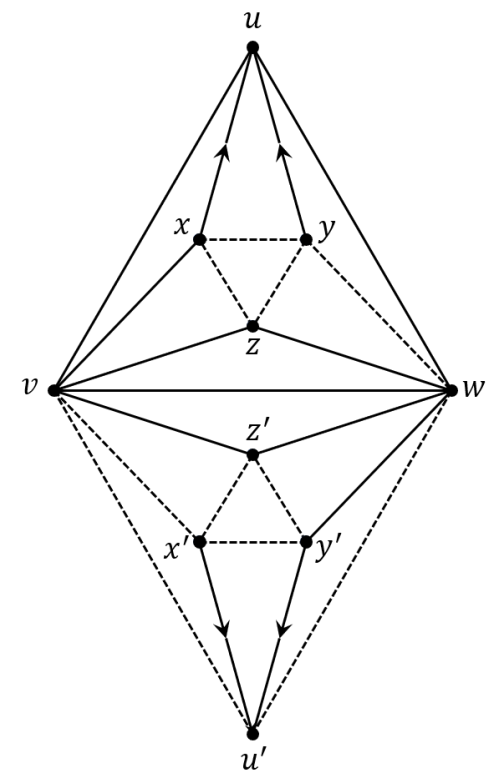

(a)

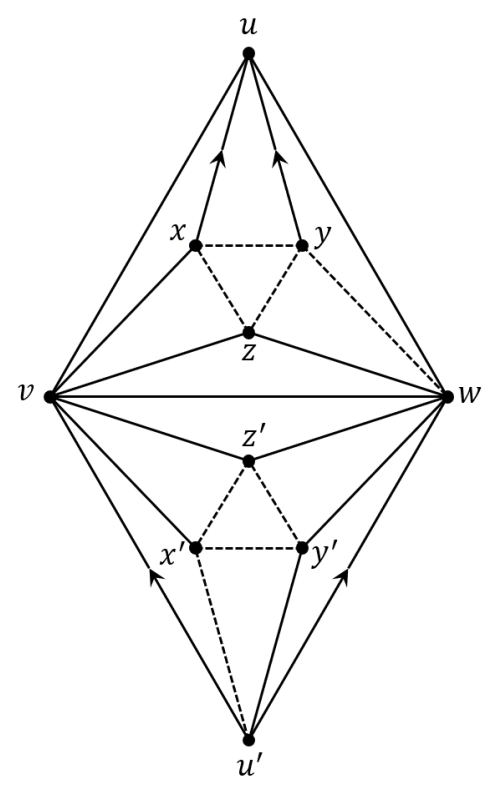

(c)

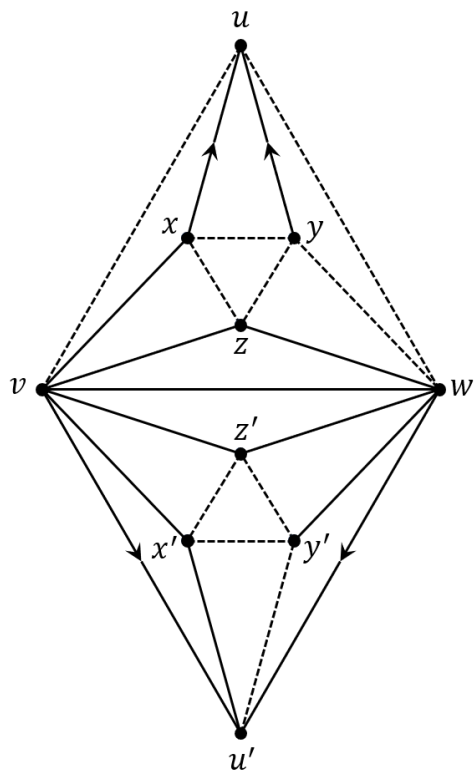

(b)

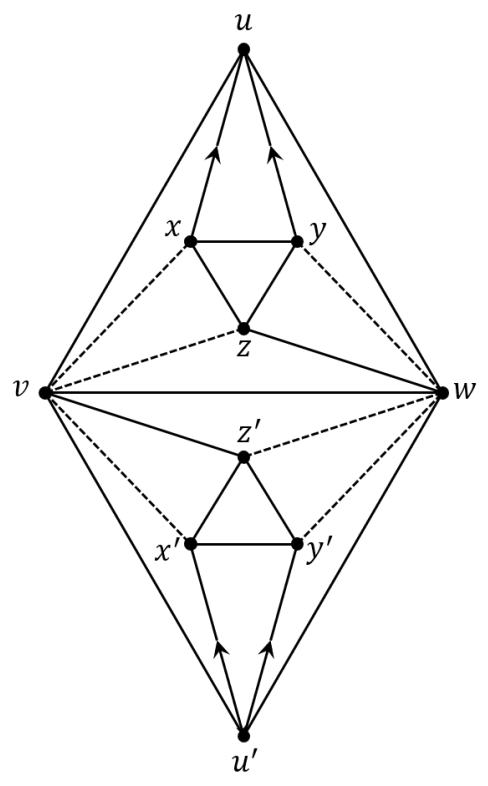

(d)

Figure 3: $S=\{(12),(34),(1234)\}$, a directed edge $e$ has $\sigma(e)=(1234)$, a solid edge $e$ has $\sigma(e)=(12)$ and a dashed edge $e$ has $\sigma(e)=(34)$. (a) $a=b=1$, (b) $a=1, b=3$, (c) $a=1, b=4$, (d) $a=3, b=4$. 
or 4 , either the triangle $x y z$ cannot be properly coloured or the triangle $x^{\prime} y^{\prime} z^{\prime}$ cannot be properly coloured.

For Figures 3(c), the permutations assigned to edges between $\left\{u, u^{\prime}\right\}$ and $\{v, w\}$ and edge $v w$ force $(\phi(v), \phi(w))=(3,4)$ or $(4,3)$. Depending on whether $(\phi(v), \phi(w))=(3,4)$ or $(4,3)$, either the triangle $x y z$ cannot be properly coloured or the triangle $x^{\prime} y^{\prime} z^{\prime}$ cannot be properly coloured.

The permutations assigned to edges in Figure $3(\mathrm{~d})$ between $\left\{u, u^{\prime}\right\}$ and $\{v, w\}$ and edge $v w$ force $(\phi(v), \phi(w))=(1,1)$ or $(2,2)$. Depending on whether $(\phi(v), \phi(w))=(1,1)$ or $(2,2)$, either the triangle $x y z$ cannot be properly coloured or the triangle $x^{\prime} y^{\prime} z^{\prime}$ cannot be properly coloured.

Next we consider the case that $S=\{(12),(34),(2314)\}$.

For $a=b=1$, or $a=1, b=3$, or $a=1, b=4$, or $a=3, b=4$, the corresponding $S$-signatures of $H_{1}$ are given in Figure 4(a), 4(b), 4(c), 4(d), respectively.

For Figures 4(a), 4(b), 4(c), the permutations assigned to edges between $\left\{u, u^{\prime}\right\}$ and $\{v, w\}$ and edge $v w$ force $(\phi(v), \phi(w))=(3,4)$ or $(4,3)$. Depending on whether $(\phi(v), \phi(w))=(3,4)$ or $(4,3)$, either the triangle $x y z$ cannot be properly coloured or the triangle $x^{\prime} y^{\prime} z^{\prime}$ cannot be properly coloured. For Figure $4(\mathrm{~d})$, the permutations assigned to edges between $\left\{u, u^{\prime}\right\}$ and $\{v, w\}$ and edge $v w$ force $(\phi(v), \phi(w))=(1,1)$ or $(2,2)$. Depending on whether $(\phi(v), \phi(w))=(1,1)$ or $(2,2)$, either the triangle $x y z$ cannot be properly coloured or the triangle $x^{\prime} y^{\prime} z^{\prime}$ cannot be properly coloured.

Finally we consider the case $S=\{(12),(34),(23)(14)\}$.

For $a=b=1$, or $a=1, b=3$, or $a=1, b=4$, or $a=3, b=4$, the corresponding $S$-signatures of $H_{1}$ are given in Figure 5(a), 5(b), 5(c), 5(d), respectively.

In Figures 5(a), the permutations assigned to edges between $\left\{u, u^{\prime}\right\}$ and $\{v, w\}$ and edge $v w$ force $(\phi(v), \phi(w))=(3,4)$ or $(4,3)$.

In Figures 5(b) and 5(c), the permutations assigned to edges between $\left\{u, u^{\prime}\right\}$ and $\{v, w\}$ and edge $v w$ force $(\phi(v), \phi(w))=(2,2)$ or $(3,3)$.

In Figures 5(d), the permutations assigned to edges between $\left\{u, u^{\prime}\right\}$ and $\{v, w\}$ and edge $v w$ force $(\phi(v), \phi(w))=(1,1)$ or $(2,2)$.

In all these cases, either the triangle $x y z$ cannot be properly coloured or the triangle $x^{\prime} y^{\prime} z^{\prime}$ cannot be properly coloured.

Case 2. $S \in\{\{(12),(34),(23)\},\{(12),(13),(234)\}\}$.

In this case, by symmetry, it suffices to consider the cases that $a=b=1$ or $a=1, b=3$ or $a=1, b=4$ or $a=b=2$ or $a=2, b=3$ or $a=3, b=4$. Note that if $S=\{(12),(34),(23)\}$, then $a=2, b=4$ is symmetric to $a=1, b=3$, and if $S=\{(12),(13),(234)\}$, then $a=2, b=4$ is symmetric to $a=3, b=4$.

For $S=\{(12),(34),(23)\}$, the corresponding $S$-signatures are given in Figures $6(a)$, $6(\mathrm{~b}), 6(\mathrm{c}), 6(\mathrm{~d}), 6(\mathrm{e})$ and $6(\mathrm{f})$, respecitvely.

The vertices $v, w$ are forced to be coloured $(\phi(v), \phi(w))=(3,4)$ or $(4,3)$ (in Figure $6(\mathrm{a})$ and $6(\mathrm{~d}))$, or $(\phi(v), \phi(w))=(2,2)$ or $(3,3)$ (in Figure $6(\mathrm{~b})$ and $6(\mathrm{c})$ and $6(\mathrm{e})$ ), or $(\phi(v), \phi(w))=(1,2)$ or $(2,1)$ (in Figure 6(f)). Each leads a contradiction as above.

For $S=\{(12),(13),(234)\}$, the corresponding $S$-signatures are given in Figures $7($ a), 


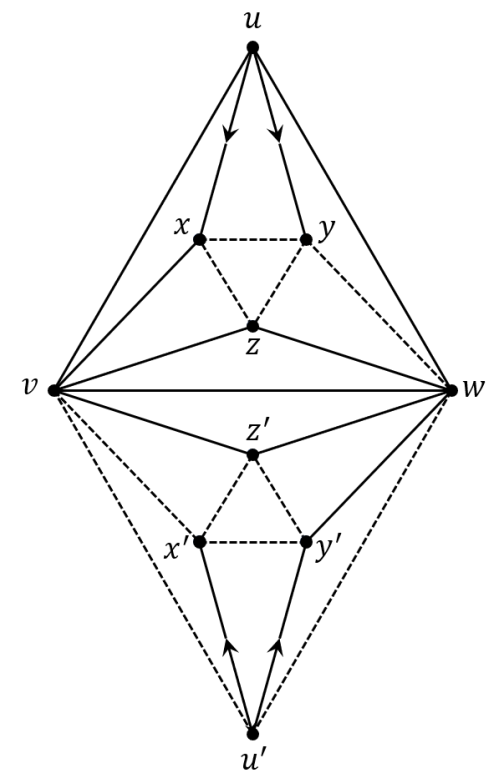

(a)

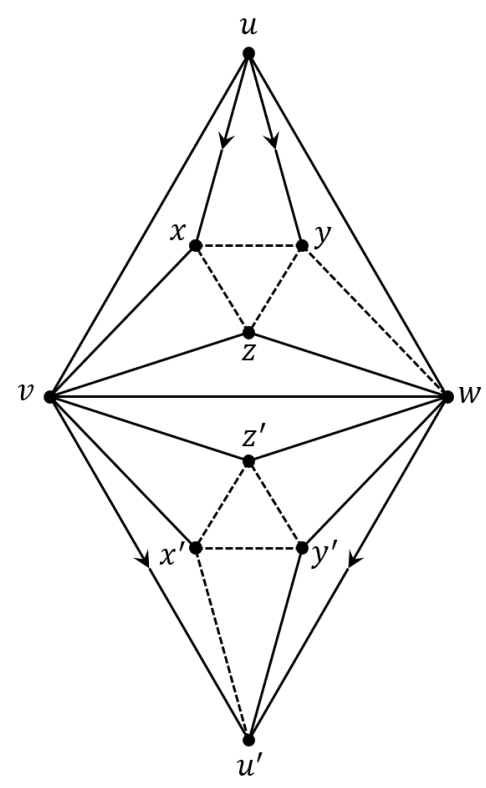

(c)

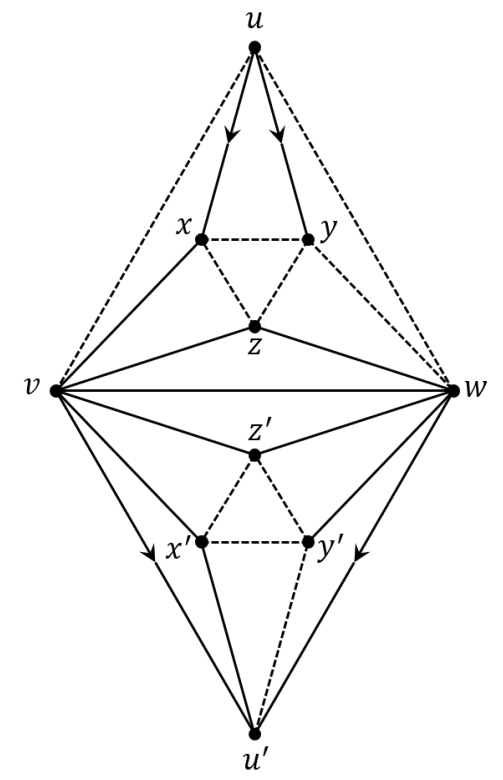

(b)

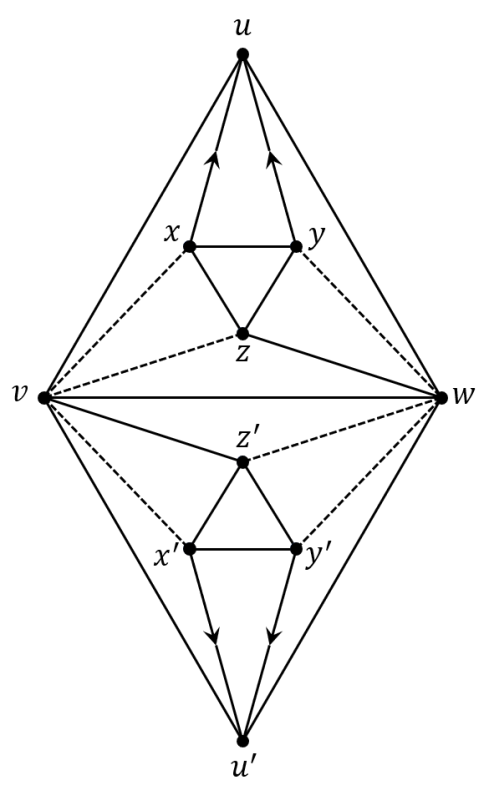

(d)

Figure 4: $S=\{(12),(34),(2314)\}$, a directed edge $e$ has $\sigma(e)=(2314)$, a solid edge $e$ has $\sigma(e)=(12)$ and a dashed edge $e$ has $\sigma(e)=(34)$. (a) $a=b=1$, (b) $a=1, b=3$, (c) $a=1, b=4$, (d) $a=3, b=4$. 


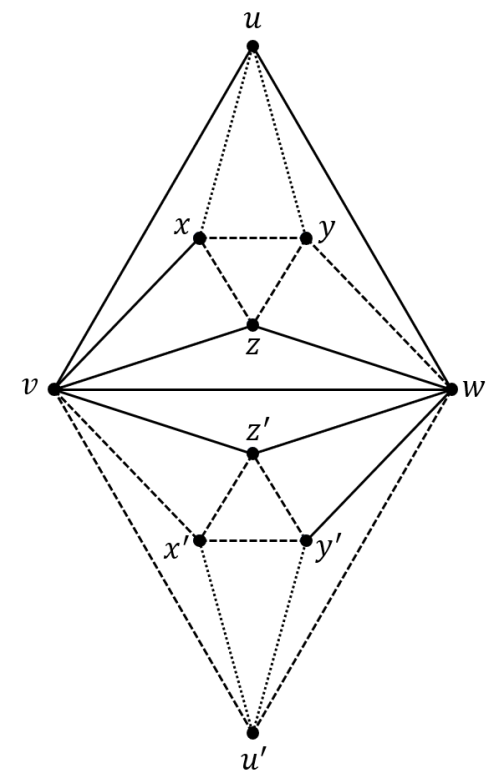

(a)

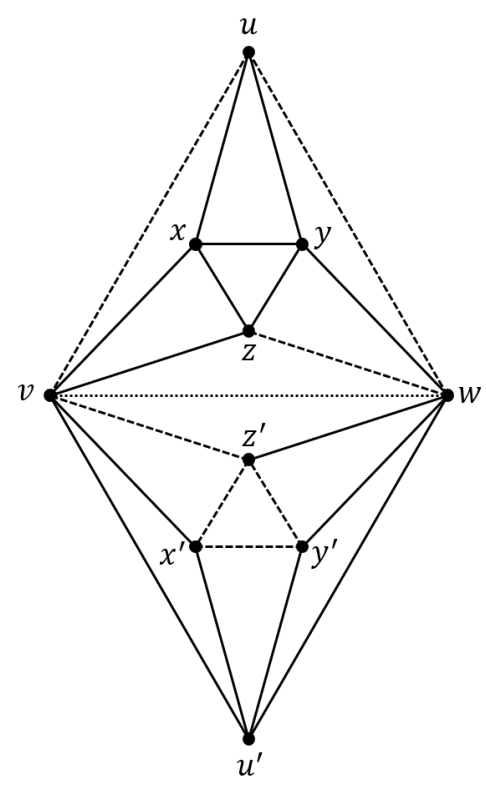

(c)

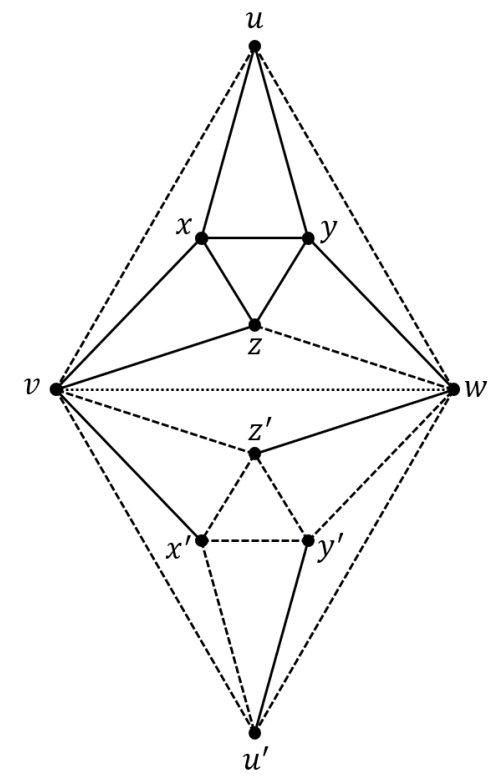

(b)

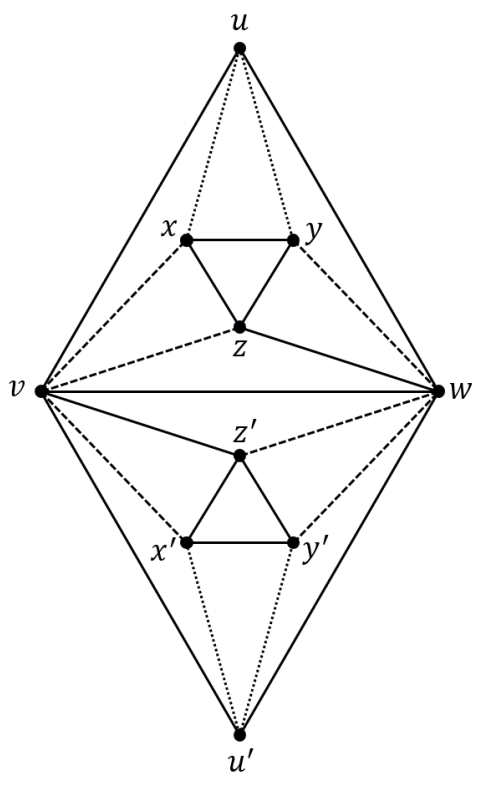

(d)

Figure 5: $S=\{(12),(34),(23)(14)\}$, a dotted edge $e$ has $\sigma(e)=(23)(14)$, a dashed edge $e$ has $\sigma(e)=(34)$ and a solid edge $e$ has $\sigma(e)=(12)$. (a) $a=b=1$, (b) $a=1, b=3$, (c) $a=1, b=4$, (d) $a=3, b=4$. 


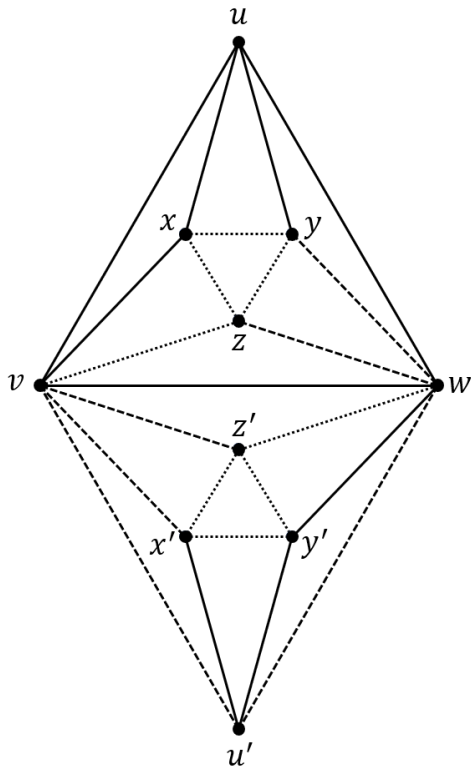

(a)

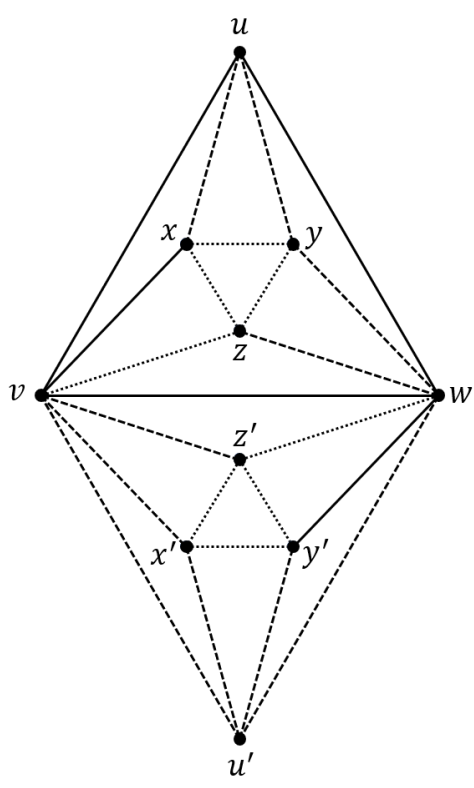

(d)

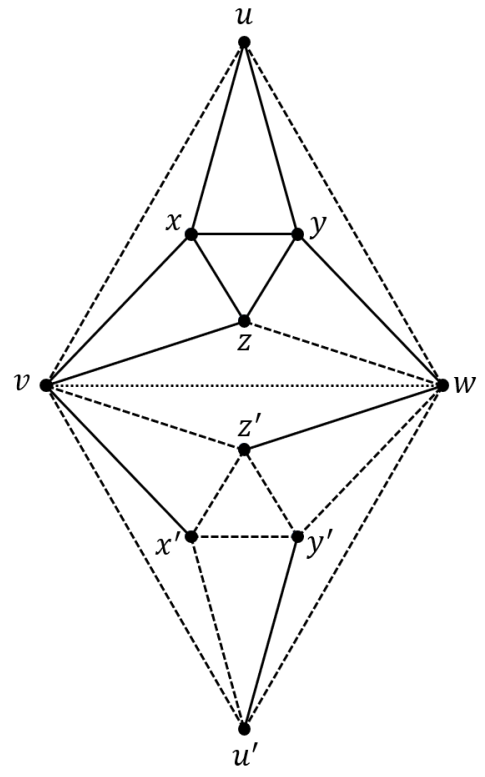

(b)

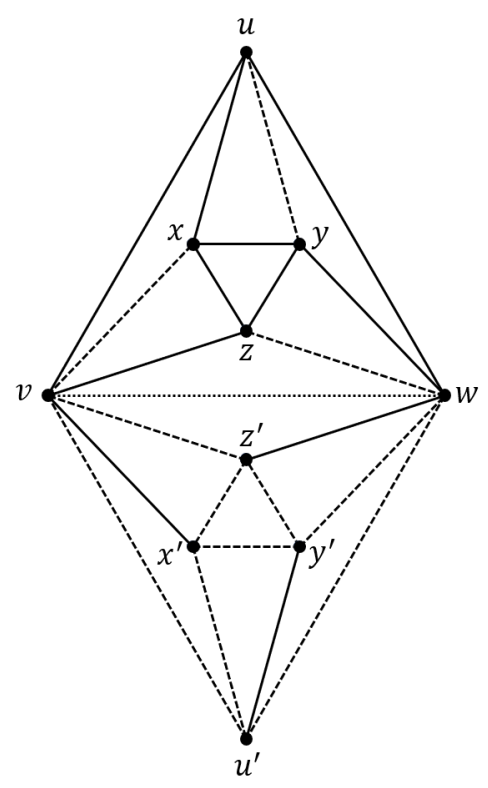

(e)

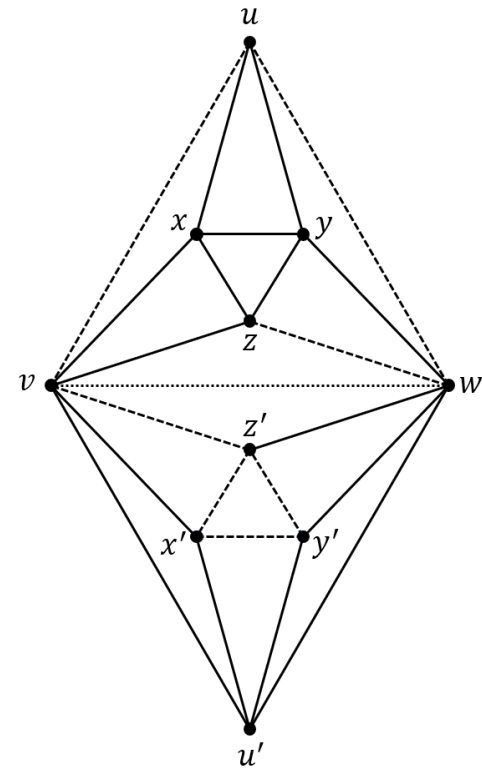

(c)

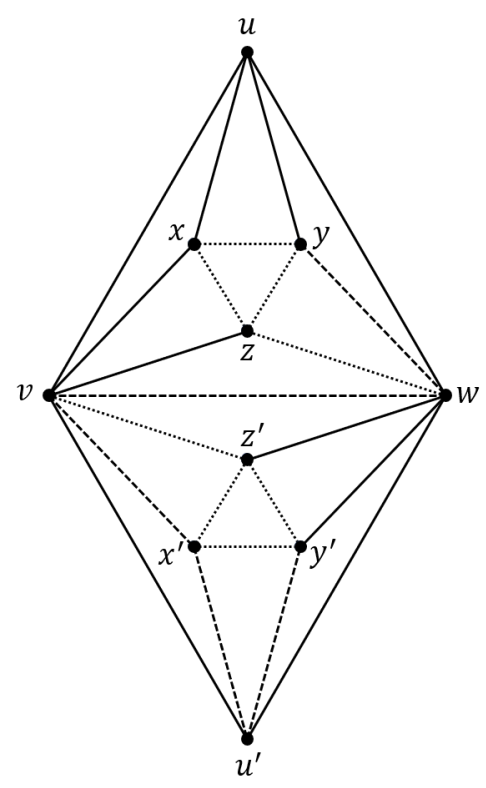

(f)

Figure 6: $S=\{(12),(34),(23)\}$, a dotted edge $e$ has $\sigma_{e}=(23)$, a dashed edge $e$ has $\sigma_{e}=(34)$ and a solid edge $e$ has $\sigma_{e}=$ (12). (a) $a=b=1$, (b) $a=1, b=3$, (c) $a=1, b=4$, (d) $a=b=2$, (e) $a=2, b=3$, (f) $a=3, b=4$. 
$7(\mathrm{~b}), 7(\mathrm{c}), 7(\mathrm{~d}), 7(\mathrm{e})$ and $7(\mathrm{f})$, respecitvely.

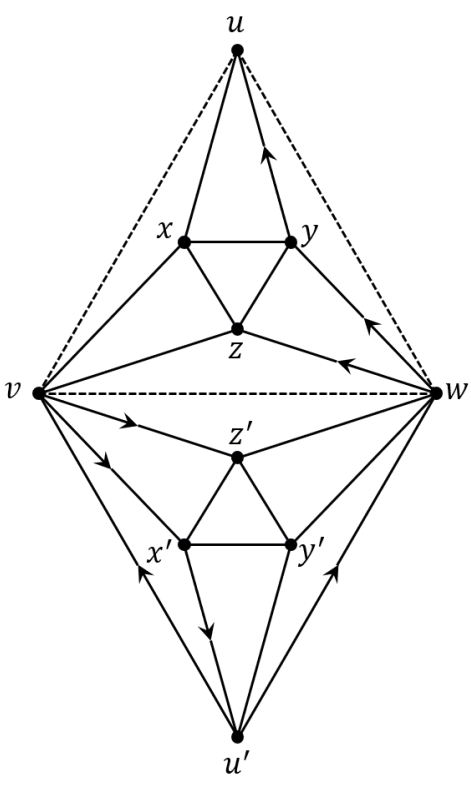

(a)

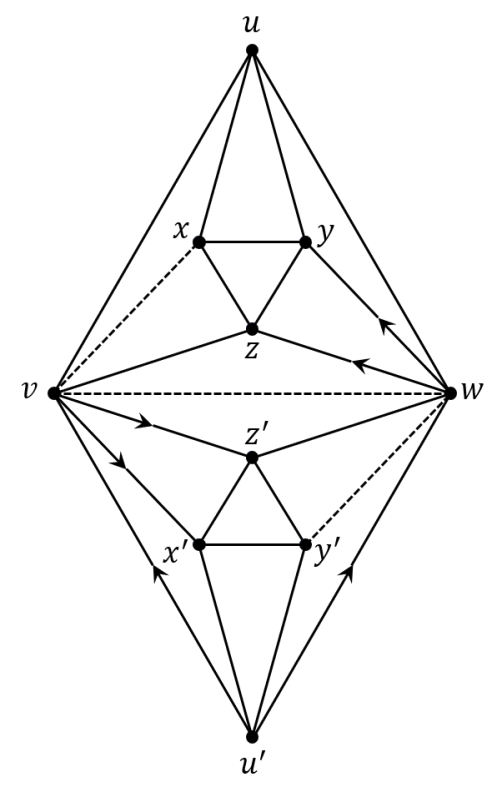

(d)

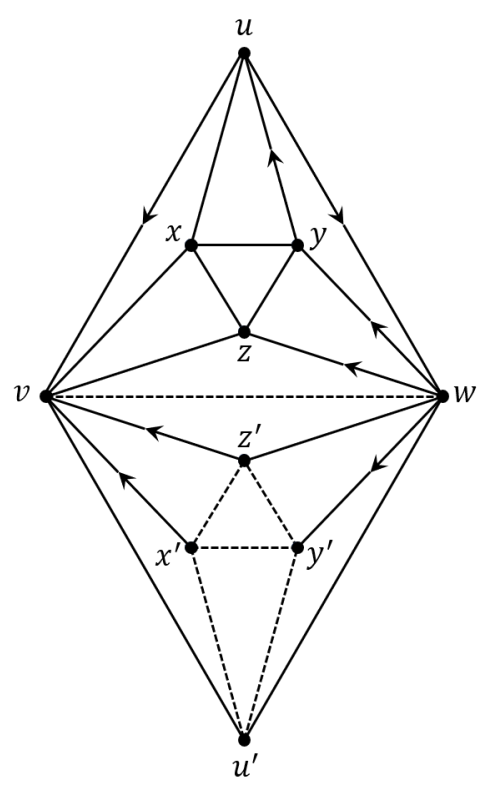

(b)

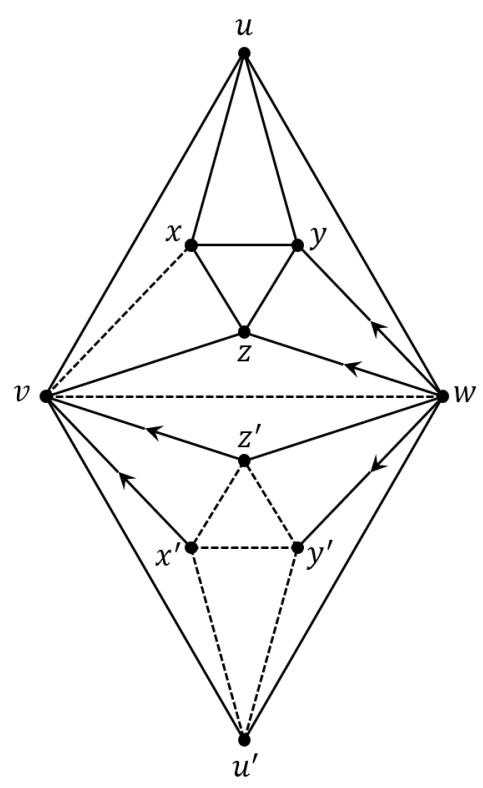

(e)

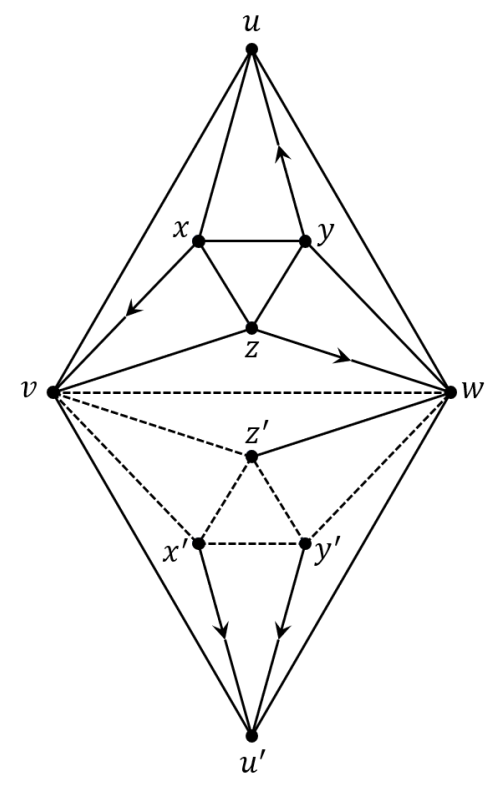

(c)

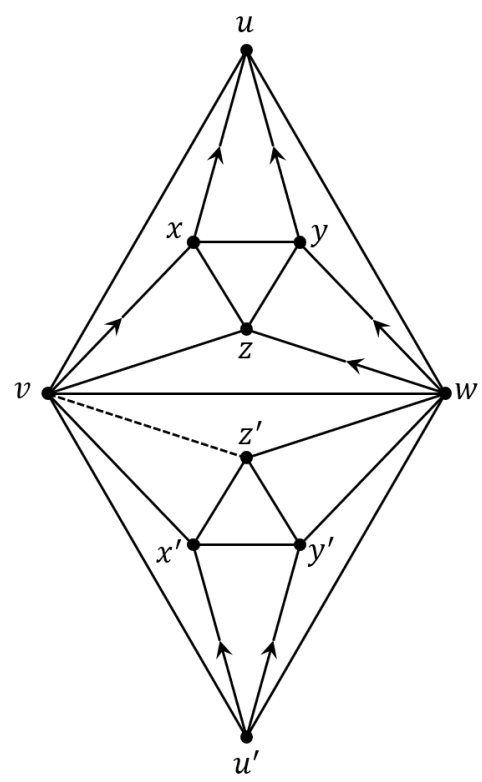

(f)

Figure 7: $S=\{(12),(13),(234)\}$, a directed edge $e$ has $\sigma_{e}=(234)$, an undirected dashed edge $e$ has $\sigma_{e}=(13)$ and a undirected solid edge $e$ has $\sigma_{e}=(12)$. (a) $a=b=1$, (b) $a=1, b=3$, (c) $a=1, b=4$, (d) $a=b=2$, (e) $a=2, b=3$, (f) $a=3, b=4$

The vertices $v, w$ are forced to be coloured $(\phi(v), \phi(w))=(2,4)$ or $(4,2)$ (in Figures $7(\mathrm{a}), 7(\mathrm{~b}), 7(\mathrm{~d})$ and $7(\mathrm{e}))$, or $(\phi(v), \phi(w))=(1,1)$ or $(3,3)$ (in Figure $7(\mathrm{c})$ ), or 
$(\phi(v), \phi(w))=(1,1)$ or $(2,2)$ (in Figure $7(\mathrm{f}))$. Each leads a contradiction as above.

Case 3. $S=\{(12),(134),(234)\}$.

In this case, by symmetry, it suffices to consider the cases that $a=b=1$ or $a=1, b=3$ or $a=3, b=4$.

For these cases, the corresponding $S$-signatures are given in Figures $8(\mathrm{a}), 8(\mathrm{~b})$ and $8(\mathrm{c})$, respecitvely.

The vertices $v, w$ are forced to be coloured $(\phi(v), \phi(w))=(1,1)$ or $(2,2)$ (in Figure 8(a), 8(b), 8(c)). Each leads a contradiction as above.

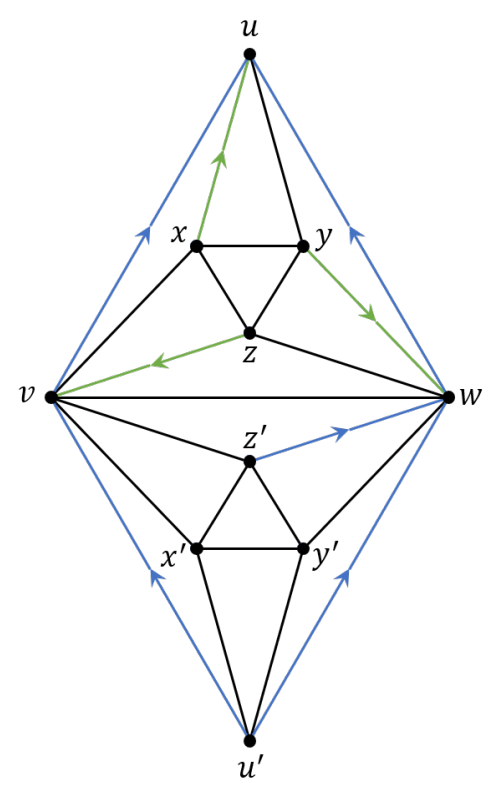

(a)

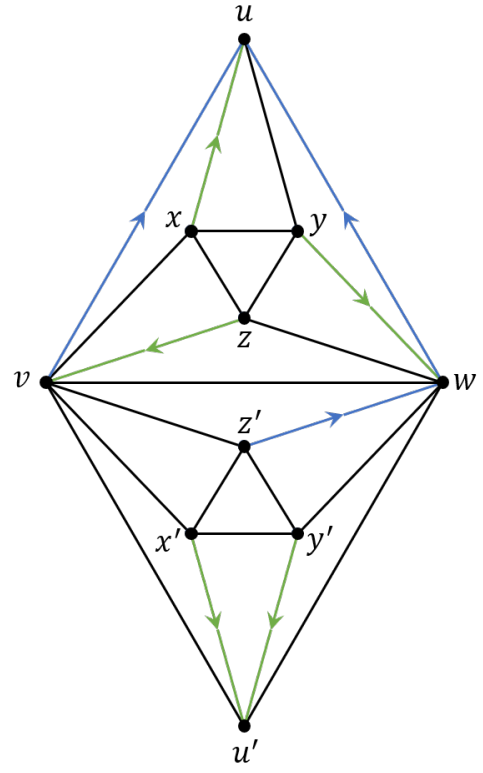

(b)

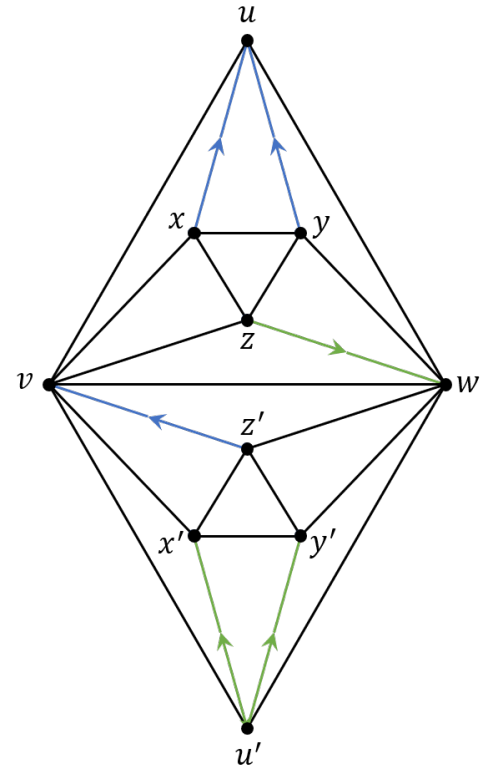

(c)

Figure 8: $S=\{(12),(134),(234)\}$, a black undirected edge $e$ has $\sigma_{e}=(12)$, a directed blue edge $e$ has $\sigma_{e}=(134)$, and a directed green edge $e$ has $\sigma_{e}=(234)$. (a) $a=b=1$, (b) $a=1, b=3$, (c) $a=3, b=4$.

Claim 16. If $S=\left\{\{(12),(34),(123)\}\right.$, then for any $a, b \in[4]$ with $\pi^{*}(a) \neq b$, either there is an $S$-signature $(D, \sigma)$ of $H_{1}$ such that there is no 4-colouring $f$ of $\left(H_{1}, \sigma\right)$ with $f(u)=a$ and $f\left(u^{\prime}\right)=b$ or there is an $S$-signature $(D, \sigma)$ of $H_{2}$ such that there is no 4-colouring $f$ of $\left(H_{2}, \sigma\right)$ with $f(u)=a$ and $f\left(u^{\prime}\right)=b$.

Proof. By symmetry, it suffices to consider the cases that $a=b=1$ or $a=1, b=3$ or $a=1, b=4$ or $a=3, b=4$.

- If $a=b=1$, then let $(D, \sigma)$ be the $S$-signature of $H_{1}$ defined as in Figure $9(\mathrm{a})$.

- If $a=1, b=3$, then let $(D, \sigma)$ be the $S$-signature of $H_{1}$ defined as in Figure $9(\mathrm{~b})$. 
- If $a=1, b=4$, then let $(D, \sigma)$ be the $S$-signature of $H_{2}$ defined as in Figure $9(\mathrm{c})$.

- If $a=3, b=4$, then let $(D, \sigma)$ be the $S$-signature of $H_{2}$ defined as in Figure $9(\mathrm{~d})$.

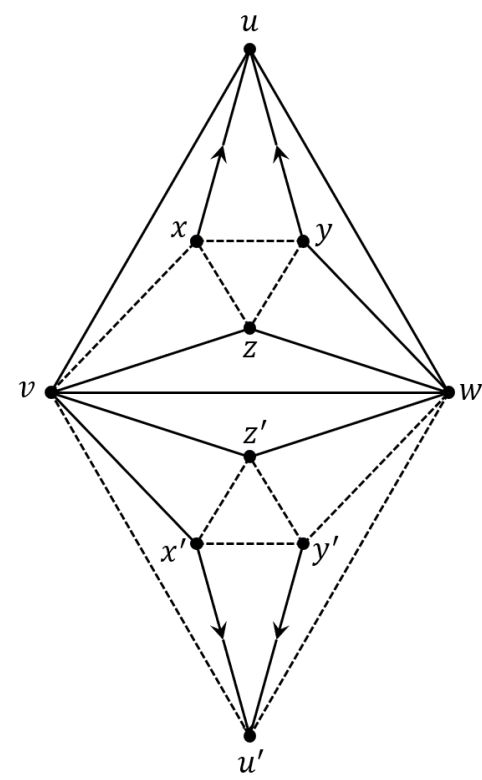

(a)

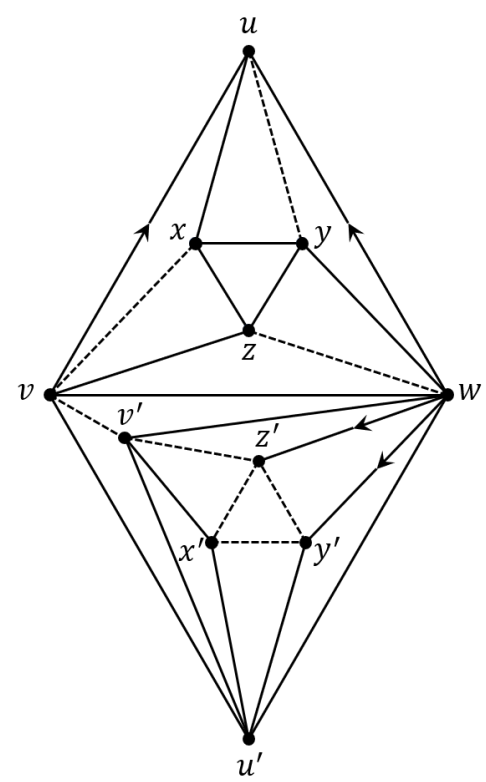

(c)

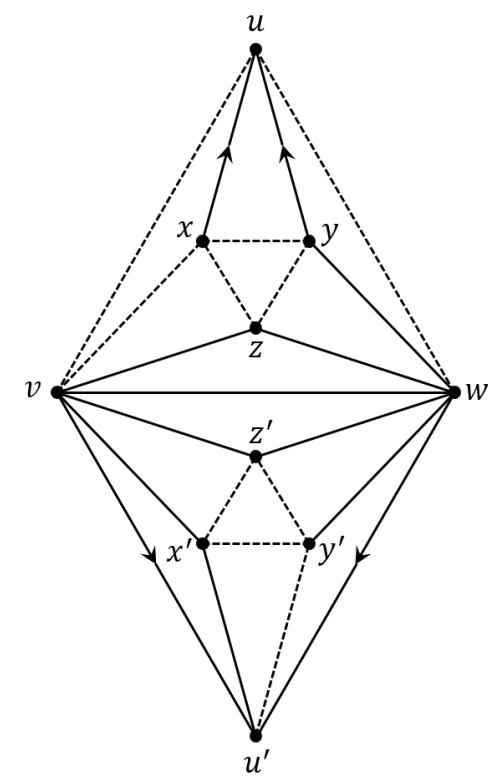

(b)

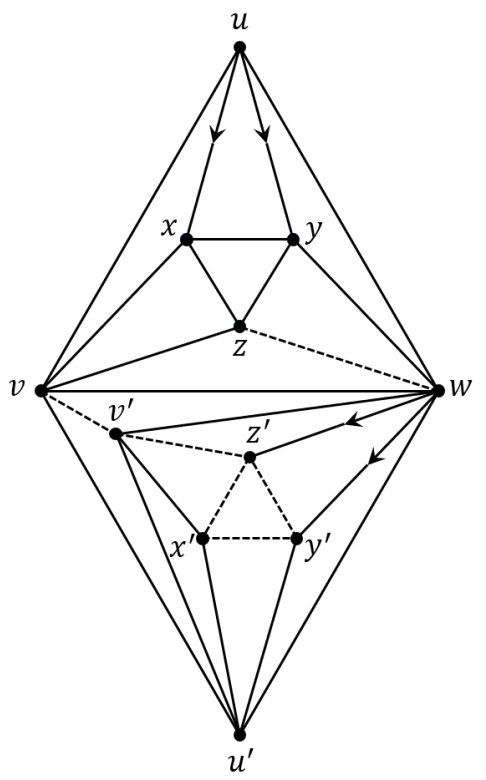

(d)

Figure 9: $S=\{(12),(34),(123)\}$, an undirected solid edge $e$ has $\sigma_{e}=(12)$, an undirected dashed edge has $\sigma_{e}=(34)$, and a directed edge $e$ has $\sigma_{e}=(123)$. (a) $a=b=1$, (b) $a=1, b=3$, (c) $a=1, b=4$, (d) $a=3, b=4$. 
Assume $\phi$ is a colouring of $\left(H_{1}, \sigma\right)$ with $\phi(u)=\phi\left(u^{\prime}\right)=1$ for the graph in Figure 9(a), or $\phi(u)=1$ and $\phi\left(u^{\prime}\right)=3$ for the graph in Figure $9(\mathrm{~b})$, or $\phi(u)=1$ and $\phi\left(u^{\prime}\right)=4$ for the graph in Figure $9(\mathrm{c})$, or $\phi(u)=3$ and $\phi\left(u^{\prime}\right)=4$ for the graph in Figure $9(\mathrm{~d})$.

The permutations on the edges $u v, u w, u^{\prime} v, u^{\prime} w$ in both of Figure 9(a) and 9(b) lead to $\phi(v)=3, \phi(w)=4$ or $\phi(v)=4, \phi(w)=3$. This implies that there are only two idenditical colours, 1 and 2, left for either the triangle $x y z$ or $x^{\prime} y^{\prime} z^{\prime}$, which is a contradiction.

The permutations on the edges $u v, u w, u^{\prime} v, u^{\prime} w$ in both of Figure $9(\mathrm{c})$ and $9(\mathrm{~d})$ lead to $\phi(v)=\phi(w)=1$ or $\phi(v)=\phi(w)=2$. In the former case (resp. the later case, in which the permutations on the edges $u^{\prime} v^{\prime}, v v^{\prime}, w v^{\prime}$ force $\phi\left(v^{\prime}\right)=3$ ), there are only two identical colours left for the triangle $x y z$ (resp. $x^{\prime} y^{\prime} z^{\prime}$ ), which is impossible.

Claim 17. If $S=\left\{\{(12),(13),(23)\}\right.$, then for any $a, b \in[4]$ with $\pi^{*}(a) \neq b$, either there is an $S$-signature $(D, \sigma)$ of $H_{3}$ such that there is no 4-colouring $f$ of $\left(H_{3}, \sigma\right)$ with $f(u)=a$ and $f\left(u^{\prime}\right)=b$ or there is an $S$-signature $(D, \sigma)$ of $H_{4}$ such that there is no 4-colouring $f$ of $\left(H_{4}, \sigma\right)$ with $f(u)=a$ and $f\left(u^{\prime}\right)=b$.

Proof. By symmetry, it suffices to consider the cases that $a=b=1$ or $a=1, b=3$ or $a=1, b=4$.

- If $a=b=1$, then let $(D, \sigma)$ be the $S$-signature of $H_{3}$ defined as in Figure 10(a).

- If $a=1, b=3$, then let $(D, \sigma)$ be the $S$-signature of $H_{3}$ defined as in Figure $10(\mathrm{~b})$.

- If $a=1, b=4$, then let $(D, \sigma)$ be the $S$-signature of $H_{4}$ defined as in Figure $10(\mathrm{c})$.

Assume $\phi$ is a colouring of $\left(H_{3}, \sigma\right)$ with $\phi(u)=\phi\left(u^{\prime}\right)=1$ for the graph in Figure 10(a), or $\phi(u)=1$ and $\phi\left(u^{\prime}\right)=3$ for the graph in Figure 10(b), or $\phi(u)=1$ and $\phi\left(u^{\prime}\right)=4$ for the graph in Figure 10(c).

In both of Figures 10(a) and 10(b), the permutations on the edges $u v, u w, u^{\prime} v, u^{\prime} w$ lead to $\phi(v)=1, \phi(w)=4$ or $\phi(v)=4, \phi(w)=1$. In the former case (resp. the later case), the permutations on the edges $u w^{\prime}, v w^{\prime}, w w^{\prime}$ (resp. $u^{\prime} v^{\prime}, v v^{\prime}, v^{\prime} w$ ) force $\phi\left(w^{\prime}\right)=1$ (resp. $\left.\phi\left(v^{\prime}\right)=1\right)$. Then there are only two identical colours, 1 and 4 , left for the triangle $x y z$ (resp. $x^{\prime} y^{\prime} z^{\prime}$ ), which is impossible.

In Figure 10(c), the permutations on the edges $u v, u w, u^{\prime} v, u^{\prime} w$ lead to $\phi(v)=\phi(w)=1$ or $\phi(v)=\phi(w)=3$. In the former case (resp. the later case, in which the permutations on the edges $u u^{\prime \prime}, v u^{\prime \prime}, w u^{\prime \prime}$ force $\phi\left(u^{\prime \prime}\right)=3$ ), there are only two identical colours left for the triangle $x y z$ (resp. $\left.x^{\prime} y^{\prime} z^{\prime}\right)$, which is a contradiction.

Lemma 18. Let $S=\{(123),(124),(134),(234)\}$. Then for any three distinct integers $\alpha, \beta, \gamma \in[4]$, there exists an $S$-signature $(D, \sigma)$ of $T$ such that there is no 4-colouring $\phi$ of $(T, \sigma)$ with $\phi(u)=\alpha, \phi(v)=\beta$ and $\phi\left(v^{\prime}\right)=\gamma$. 


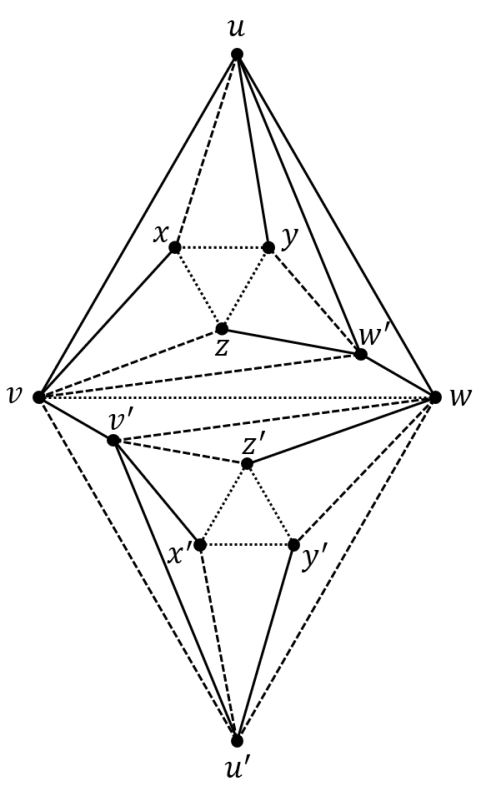

(a)

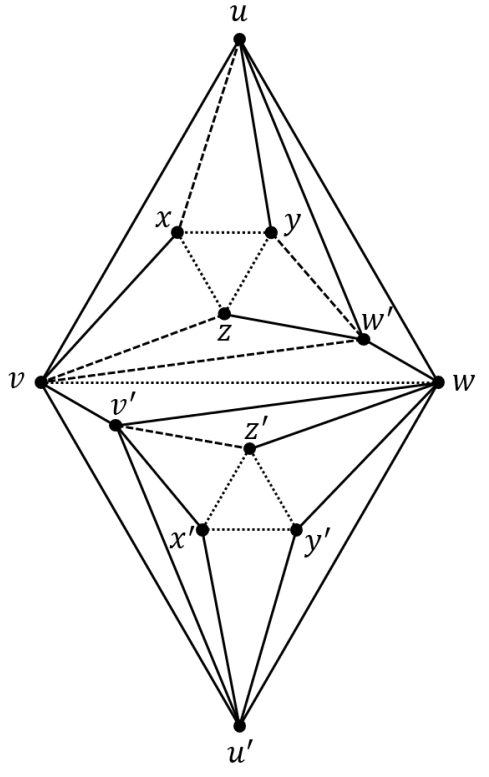

(b)

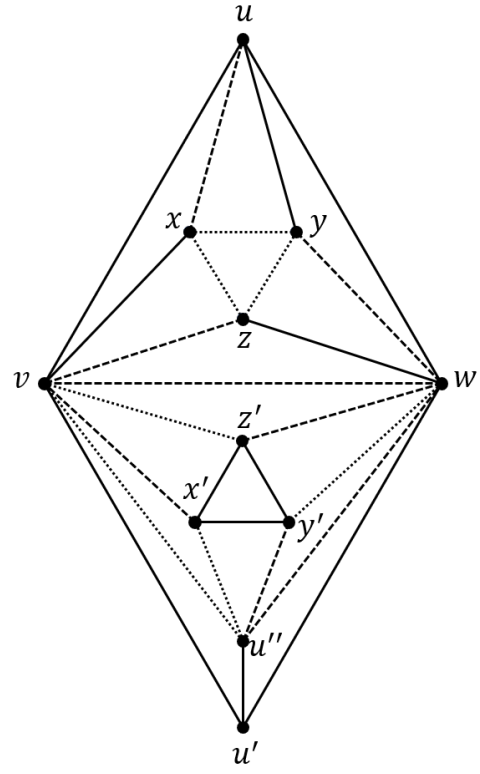

(c)

Figure 10: $S=\{(12),(13),(23)\}$, a solid edge $e$ has $\sigma_{e}=(12)$, a dashed edge $e$ has $\sigma_{e}=(13)$, and a dotted edge $e$ has $\sigma_{e}=(23)$. (a) $a=b=1$, (b) $a=1, b=3$, (c) $a=1, b=4$.

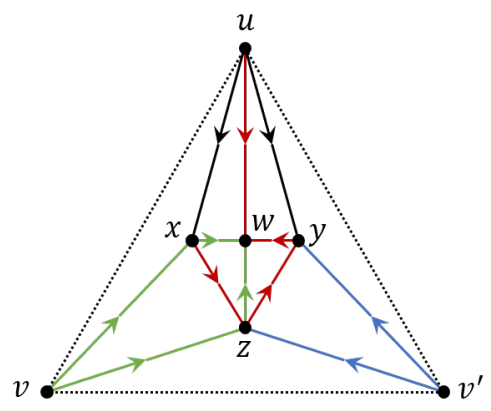

Figure 11: The $S$-signature of $T$, where a directed red edge $e$ has $\sigma(e)=(123)$, a directed blue edge $e$ has $\sigma(e)=(124)$, a directed green edge $e$ has $\sigma(e)=(134)$, and a directed black edge $e$ has $\sigma(e)=(234)$. 
Proof. By symmetry, it suffices to consider the cases that $\alpha=1, \beta=2, \gamma=3$. Let $(D, \sigma)$ be the $S$-signature of $T$ defined as in Figure 11.

Assume $\phi$ is a colouring of $(T, \sigma)$ with $\phi(u)=1, \phi(v)=2, \phi\left(v^{\prime}\right)=3$. The permutations on the edges $u x, u y, v x, v z, v^{\prime} y, v^{\prime} z$ lead to $\phi(x) \in\{3,4\}, \phi(y) \in\{2,4\}, \phi(z) \in\{1,4\}$. If $\phi(z)=1$, then $\phi(x)=\phi(y)=4$, which implies there is no colour for $w$. If $\phi(z)=4$, then $\phi(x)=3, \phi(y)=2$, which implies there is no colour for $w$, a contradiction.

Claim 19. If $S=\{(123),(124),(134),(234)\}$, then for any $a, b \in[4]$ with $\pi^{*}(a) \neq b$, there is an $S$-signature $(D, \sigma)$ of $H_{5}$ such that there is no 4-colouring $f$ of $\left(H_{5}, \sigma\right)$ with $f(u)=a$ and $f\left(u^{\prime}\right)=b$.

Proof. By symmetry, it suffices to consider the cases that $a=b=1$ or $a=3, b=4$.

- If $a=b=1$, then let $(D, \sigma)$ be the $S$-signature of $H_{5}$ defined as in Figure $12($ a).

- If $a=3, b=4$, then let $(D, \sigma)$ be the $S$-signature of $H_{5}$ defined as in Figure $12(\mathrm{~b})$.

Moreover, for the graph in Figure 12(a),

- The signature $(D, \sigma)$ of the copy of $T$ inside triangle $u v v^{\prime \prime}$ is such that there is no 4-colouring $\phi$ of $(T, \sigma)$ with $\phi(u)=1, \phi(v)=2, \phi\left(v^{\prime \prime}\right)=3$.

- The signature $(D, \sigma)$ of the copy of $T$ inside triangle $u v^{\prime} v^{\prime \prime}$ is such that there is no 4-colouring $\phi$ of $(T, \sigma)$ with $\phi(u)=1, \phi\left(v^{\prime}\right)=3, \phi\left(v^{\prime \prime}\right)=2$.

- The signature $(D, \sigma)$ of the copy of $T$ inside triangle $u^{\prime} v v^{\prime}$ is such that there is no 4-colouring $\phi$ of $(T, \sigma)$ with $\phi\left(u^{\prime}\right)=1, \phi(v)=3, \phi\left(v^{\prime}\right)=2$.

For the graph in Figure 12(b),

- The signature $(D, \sigma)$ of the copy of $T$ inside triangle $u v v^{\prime \prime}$ is such that there is no 4-colouring $\phi$ of $(T, \sigma)$ with $\phi(u)=3, \phi(v)=1, \phi\left(v^{\prime \prime}\right)=2$.

- The signature $(D, \sigma)$ of the copy of $T$ inside triangle $u v^{\prime} v^{\prime \prime}$ is such that there is no 4-colouring $\phi$ of $(T, \sigma)$ with $\phi(u)=3, \phi\left(v^{\prime}\right)=2, \phi\left(v^{\prime \prime}\right)=1$.

- The signature $(D, \sigma)$ of the copy of $T$ inside triangle $u^{\prime} v v^{\prime}$ is such that there is no 4-colouring $\phi$ of $(T, \sigma)$ with $\phi\left(u^{\prime}\right)=4, \phi(v)=2, \phi\left(v^{\prime}\right)=1$.

Consider the graph in Figure 12(a). Assume $\phi$ is a colouring of $\left(H_{5}, \sigma\right)$ with $\phi(u)=$ $\phi\left(u^{\prime}\right)=1$.

The permutations on the edges $u v, u v^{\prime}, u^{\prime} v, u^{\prime} v^{\prime}$ lead to $\phi(v)=\phi\left(v^{\prime}\right)=2$ or $\phi(v)=$ $\phi\left(v^{\prime}\right)=3$ or $\phi(v)=3, \phi\left(v^{\prime}\right)=2$. The copy of $T$ inside triangle $u^{\prime} v v^{\prime}$ implies that $\left(\phi(v), \phi\left(v^{\prime}\right)\right) \neq(3,2)$.

If $\phi(v)=\phi\left(v^{\prime}\right)=2$, the permutations on the edges $u v^{\prime \prime}, v v^{\prime \prime}, v^{\prime} v^{\prime \prime}$ force $\phi\left(v^{\prime \prime}\right)=3$. Thus the triangle $\left(u, v, v^{\prime \prime}\right)$ is coloured by colours $(1,2,3)$, a contradiction. 


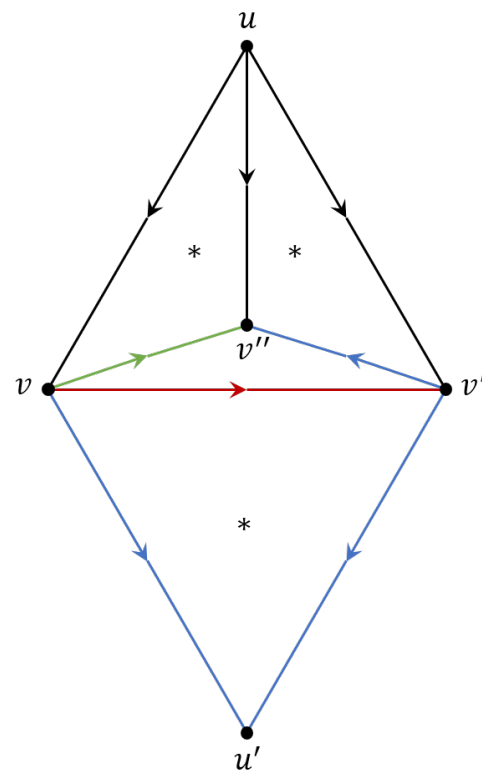

(a)

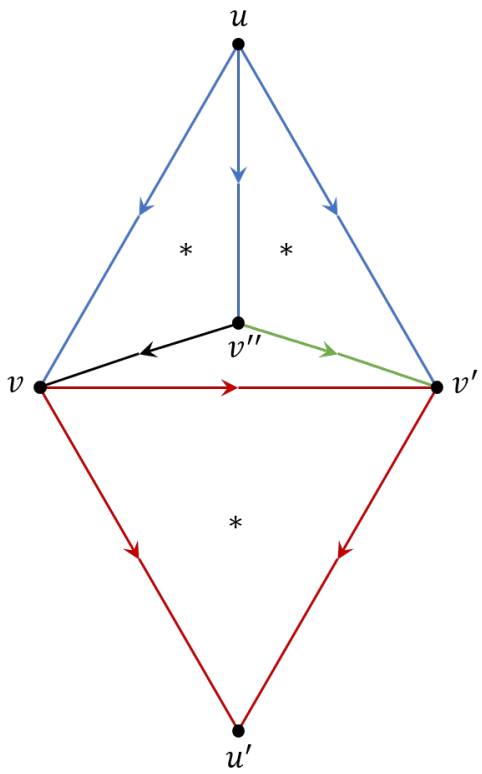

(b)

Figure 12: $S=\{(123),(124),(134),(234)\}$, a directed red edge $e$ has $\sigma_{e}=(123)$, a directed blue edge $e$ has $\sigma_{e}=(124)$, a directed green edge $e$ has $\sigma_{e}=(134)$, and a directed black edge $e$ has $\sigma_{e}=(234)$. (a) $a=b=1$ (b) $a=3, b=4$.

If $\phi(v)=\phi\left(v^{\prime}\right)=3$, then the permutations on the edges $u v^{\prime \prime}, v v^{\prime \prime}, v^{\prime} v^{\prime \prime}$ force $\phi\left(v^{\prime \prime}\right)=2$. Thus the triangle $\left(u, v^{\prime}, v^{\prime \prime}\right)$ is coloured by colours $(1,3,2)$, a contradiction.

For the graph in Figure 12(b), assume $\phi$ is a colouring of $\left(H_{5}, \sigma\right)$ with $\phi(u)=3$ and $\phi\left(u^{\prime}\right)=4$.

The permutations on the edges $u v, u v^{\prime}, u^{\prime} v, u^{\prime} v^{\prime}$ lead to $\phi(v)=\phi\left(v^{\prime}\right)=1$ or $\phi(v)=$ $\phi\left(v^{\prime}\right)=2$ or $\phi(v)=2, \phi\left(v^{\prime}\right)=1$.

The copy of $T$ inside triangle $u^{\prime} v v^{\prime}$ implies that $\left(\phi(v), \phi\left(v^{\prime}\right)\right) \neq(2,1)$.

If $\phi(v)=\phi\left(v^{\prime}\right)=1$, then the permutations on the edges $u v^{\prime \prime}, v v^{\prime \prime}, v^{\prime} v^{\prime \prime}$ force $\phi\left(v^{\prime \prime}\right)=2$, a contradiction (as the copy of $T$ inside triangle $u v v^{\prime \prime}$ forbids such a colouring).

If $\phi(v)=\phi\left(v^{\prime}\right)=2$, then the permutations on the edges $u v^{\prime \prime}, v v^{\prime \prime}, v^{\prime} v^{\prime \prime}$ force $\phi\left(v^{\prime \prime}\right)=1$, a contradiction (as the copy of $T$ inside triangle $u v^{\prime} v^{\prime \prime}$ forbids such a colouring).

Combining Claims 15, 16, 17 and 19, we complete the proof of Lemma 14.

\section{References}

[1] Z. Dvořák and L. Postle. List-coloring embedded graphs without cycles of lengths 4 to 8. arXiv: 1508.03437, 2015.

[2] F. Jaeger, N. Linial, C. Payan, and M. Tarsi. Group connectivity of graphs - A non-homongenous analogue of nowhere-zero flow. J. Combin. Theory Ser. B, 56: 165-182, 1992. 
[3] L. Jin, T. Wong and X. Zhu. Colouring of generalized signed planar graphs. arXiv:1811.08584, 2019.

[4] Y. Kang and E. Steffen. The chromatic spectrum of signed graphs. Discrete Math., 339: 2660-2663, 2016.

[5] Y. Kang and E. Steffen. Circular coloring of signed graphs. J. Graph Theory, 87(2): 135-148, 2018.

[6] D. Král, O. Pangrác and H. J. Voss. A note on group coloring. J. Graph Theory, 50(2): 123-129, 2005.

[7] H. Lai, X. Li, Y. Shao and M. Zhan. Group Connectivity and Group Colorings of Graphs - A Survey. Acta Mathematica Sinica, English Series, 27(3): 405-434, 2011.

[8] E. Máčajová, A. Raspaud and M. Škoviera. The chromatic number of a signed graph. Electron. J. Combin. 23(1): 1-14, 2016.

[9] M. Mirzakhani. A small non-4-choosable planar graph. Bull. Inst. Combin. Appl., 17: 15-18, 1996.

[10] M. Montassier. A small non- $Z_{4}$-colourable planar graph. Discrete Math., 307: 16841686, 2007.

[11] F. Kardoš and J. Narboni. On the 4-color theorem for signed graph. arXiv:1906.05638, 2019.

[12] T. Zaslavsky. Signed graph coloring. Discrete Math., 39: 215-228, 1982.

[13] T. Zaslavsky. Biased graphs III, Chromatic Invariants. J. Combin. Theory Ser. B, 64(1): 17-88, 1995.

[14] X. Zhu. A refinement of choosability of graphs. J. Combin. Theory Ser. B, 143-164, 2020 . 